Preprint typeset in JHEP style - HYPER VERSION

\title{
Shockwaves and deep inelastic scattering within the gauge/gravity duality
}

\author{
E. Avsar, ${ }^{a}$ E. Iancu, ${ }^{a}$ L. McLerran ${ }^{b}$ and D.N. Triantafyllopoulos ${ }^{c}$ \\ ${ }^{a}$ Institut de Physique Théorique de Saclay, F-91191 Gif-sur-Yvette, France \\ ${ }^{b}$ RIKEN BNL Research Center and Physics Department, Brookhaven National Laboratory, \\ Upton, NY 11973, USA \\ ${ }^{c}$ Institut für Theoretische Physik, Technische Universität Wien, 1040 Vienna, Austria \\ E-mail: Emil.Avsar@cea.fr, Edmond.Iancu@cea.fr, mclerran@bnl.gov, \\ dionysis@hep.itp.tuwien.ac.at
}

\begin{abstract}
Within the gauge/gravity correspondence, we discuss the general formulation of the shockwave metric which is dual to a 'nucleus' described by the strongly-coupled $\mathcal{N}=4$ SYM theory in the limit where the number of colors $N_{c}$ is arbitrarily large. We emphasize that the 'nucleus' must possess $N_{c}^{2}$ degrees of freedom per unit volume, so like a finite-temperature plasma, in order for a supergravity description to exist. We critically reassess previous proposals for introducing transverse inhomogeneity in the shockwave and formulate a new proposal in that sense, which involves no external source but requires the introduction of an 'infrared' cutoff which mimics confinement. This cutoff however plays no role when the shockwave is probed by a highly virtual projectile, so like in deep inelastic scattering. We consider two such projectiles, the dilaton and the $\mathcal{R}$-current, and compute the respective structure functions including unitarity corrections. We find that there are no leading-twist contributions to the structure functions at high virtuality, meaning that there are no point-like constituents in the strongly coupled 'nucleus'. In the black-disk regime at low virtuality, the structure functions are suggestive of parton saturation with occupation numbers of order one. The saturation momentum $Q_{s}$ grows with the energy like $Q_{s}^{2} \sim 1 / x$ (with $x$ the Bjorken variable), which is the hallmark of graviton exchanges and is also necessary for the fulfillment of the energy-momentum sum rules.
\end{abstract}




\section{Contents}

1. Introduction 1

2. Shockwaves with impact parameter dependence in $\mathbf{A d S}_{5}$

2.1 Type-I metric: no source in the bulk 8

2.2 Type-II metric: pointlike source in the bulk 12

3. DIS off the shockwave: the dilaton 14

3.1 Formalism and structure function 14

3.2 Eikonal scattering 18

3.3 From single scattering to the saturation momentum 21

3.4 Structure function at saturation 24

4. DIS off the shockwave: the $\mathcal{R}$-current 26

4.1 General equations: from the 5D action to the polarization tensor 26

4.2 The classical solution 29

4.3 Structure functions and their partonic interpretation 30

A. From black holes to shockwaves 35

B. The bulk-to-bulk propagator 36

G. Prefactor of the structure function 38

D. Explicit check of sum rules 39

\section{Introduction}

Some of the experimental discoveries at RHIC, notably the unexpectedly large medium effects known as elliptic flow and jet quenching, led to the suggestion that the deconfined hadronic matter produced in the intermediate stages of a heavy ion collision might be strongly interacting $[1,2]$. This observation triggered a large theoretical activity aiming at understanding the properties of a strongly-coupled plasma via string theory methods, within the AdS/CFT correspondence (see, e.g., the review papers [3-5] and refs. therein). Part of this activity concentrated on the 'dual' formulation of a heavy ion collision at strong coupling. The main motivation for that problem is less in the description of the nuclear wavefunctions by themselves - a real ultrarelativistic nucleus in QCD is rather described via weak coupling methods, because of the high parton density produced by the high-energy evolution [6] - , but rather in understanding the late stages of the collision and, especially, study thermalization. If the deconfined matter produced after a heavy ion collision is effectively strongly coupled, then one may hope that the 
subsequent dynamics leading to thermalization is not very sensitive to the perturbative early stages, and thus it can be studied by simulating a full collision in AdS/CFT. Such a study may help explaining one of the main puzzles left by RHIC, which is the very short thermalization time suggested by the elliptic flow data.

The AdS/CFT correspondence [7-9] deals with the conformally symmetric $\mathcal{N}=4$ supersymmetric Yang-Mills (SYM) theory, and not directly with QCD. But thus is probably not a major impediment so long as we are interested in the temperature range $2 T_{c} \lesssim T \lesssim 5 T_{c}$ (the relevant range for heavy ion experiments at RHIC and LHC), where the QCD plasma itself appears to be nearly conformal, as shown by lattice studies [10]. For practical reasons, all the studies so far were limited to the 'supergravity approximation' valid when the number of colors $N_{c}$, and hence also the 't Hooft coupling $\lambda=g^{2} N_{c}$, are arbitrarily large. (The gauge coupling $g$ is assumed to be small, $g \ll 1$, but fixed.) In this approximation, a plasma or a nucleus are described as modifications of the asymptotically $\mathrm{AdS}_{5}$ geometry that the string theory lives in, and the dynamics reduces to classical gravity in this curved space-time. In order for the respective metric perturbation to survive as $N_{c} \rightarrow \infty$, one needs to assume that the system under consideration possesses $N_{c}^{2}$ degrees of freedom per unit volume. This is indeed the case for the $\mathcal{N}=4 \mathrm{SYM}$ plasma at finite temperature, whose dual description is a black-brane in $\mathrm{AdS}_{5}$ [11] but it would not be true for a QCD-like nucleus, which is built with colorless 'hadrons'.

Still, if we are merely interested in studying thermalization, then one can replace the colliding nuclei with finite-size slices of plasma, represented as slices of 'black-holes' (more properly, black branes) in $\mathrm{AdS}_{5}$. In the center-of-mass frame, where these 'nuclei' (prior to the collision) are highly energetic and hence strongly Lorentz contracted, the black-hole slices appear as shockwaves directing against each other. The ultimate objective is to explicitly compute the scattering between two such shockwaves by solving Einstein equations, and thus investigate the evolution towards a black brane at late stages, which is synonymous of thermalization. Several steps have been already done in that sense, with interesting results, and the dynamical formation of a black hole has been already seen within supergravity calculations [12-21].

But before attacking this complicated problem, it is the very formulation of the gravity dual of a nucleus as a shockwave which still meets with difficulties in the literature. The would-be natural recipe for building such a shockwave in view of the discussion above - namely, start with a slice of the plasma with finite longitudinal width $L \gg 1 / T$ in the plasma rest frame and then boost the dual 'black-hole slice' metric up to a large Lorentz factor $\gamma \gg 1$ - is not fully satisfactory at a conceptual level, since a 'slice of a black-hole' in $\mathrm{AdS}_{5}$ is not an exact solution to Einstein equations. Yet, as we shall demonstrate in Appendix A, this approximate procedure has the merit to generate a shockwave metric which is similar to the one that we shall propose via different considerations, in Sect. 2.

An important related problem is that of the conformal symmetry breaking: this was already necessary in order to built hadronic-like bound states in the $\mathcal{N}=4$ SYM theory [22-26], and one can hardly see how one could construct a nuclear shockwave without introducing any scale in the problem. Yet, the original proposal in that sense, due to Janik and Peschanski [12], seems not to require any such a scale. As we shall argue in Sect. 2, such a scale must nevertheless be added by hand to that construction, in the form of a cutoff on the radial dimension ${ }^{1}$ of $\mathrm{AdS}_{5}$

\footnotetext{
${ }^{1}$ Throughout this paper, we will be using Fefferman-Graham coordinates (see Sect. 2 for precise definitions),
} 
either a 'hard wall', i.e. a sharp radial cutoff at $z=1 / \Lambda$ [23], or a 'soft wall', like in Ref. [25]. This cutoff corresponds to an infrared cutoff $\sim \Lambda$ in the $\mathcal{N}=4$ SYM theory and mimics the confinement physics of QCD. Incidentally, the metric produced by boosting a slice of the plasma is identical to that by Janik and Peschanski near the Minkowski boundary (for $z \ll 1 / T$ ), but it naturally involves a radial cutoff, namely the black-hole horizon at $z \sim 1 / T$ (see Appendix A).

The need for a radial cutoff in $\mathrm{AdS}_{5}$ can be also seen via a different argument, which is internal to the supergravity construction: without such a cutoff, the shockwave metric develops a genuine space-time singularity at $z \rightarrow \infty$, and not just a coordinate singularity. This has been recognized in the literature [27] for the analog of the Janik-Peschanski metric in 4 dimensions, known as the Kaigorodov space-time [28], but a similar argument holds in 5 dimensions. The argument is quite subtle, since the singularity at $z \rightarrow \infty$ does not show up in any of the curvature scalars computed from this metric, but is of a more general type, known as $g$-incompleteness [29].

The issue of the radial cutoff becomes even sharper when trying to introduce transverse inhomogeneity in the shockwave, corresponding to a profile for the nuclear matter distribution in the plane transverse to the collision axis. The two types of proposals in that sense that we are aware of $[14-17,30]$ use external sources to break down conformal symmetry. Besides the difficulty to motivate the $N_{c}^{2}$-scaling for the strength of these sources, these proposals have some unwanted features, which make them unrealistic from the viewpoint of QCD. The proposal in Ref. [14] uses a pointlike source located in the bulk of $\mathrm{AdS}_{5}$ to produce a 'nuclear' energymomentum distribution which falls off at large transverse distances according to a power law, instead of the exponential law expected in the presence of confinement. Accordingly, when used for scattering problems, this shockwave yields cross-sections which violate the Froissart bound. The proposal in Ref. [30] is even more objectionable, in that one is mistreating the 'ultraviolet' (high-momentum) part of the 'nuclear' wavefunction. This proposal involves a source on the boundary of $\mathrm{AdS}_{5}$, hence the corresponding metric perturbation does not vanish when approaching the boundary. Via the UV/IR correspondence [26,31-35], this means that the spectrum of the quantum modes included in the dual 'nuclear' wavefunction is flat in the high-momentum limit, rather than rapidly falling down, as it should on physical grounds.

On the other hand, the analysis in Ref. [30] has the virtue to have identified a class of exact solutions to Einstein equations of the shockwave type, which allow for a generic inhomogeneity in the transverse plane. This analysis lies at the basis of our shockwave proposal in this paper. One can succinctly describe our proposal as follows: among the two general solutions found in [30] and which involves the modified Bessel functions $\mathrm{K}_{2}$ and, respectively, $\mathrm{I}_{2}$, we shall discard the solution $\propto \mathrm{K}_{2}$ that was adopted in Ref. [30] and keep the other solution $\propto \mathrm{I}_{2}$. The latter is the generalization of the shockwave metric by Janik and Peschanski [12] (which is homogeneous in the transverse plane) to a generic transverse energy-momentum distribution. This solution vanishes near the boundary $(z \rightarrow 0)$ like $z^{4}$, meaning that the spectrum of the modes included in the dual 'nucleus' has an acceptable high-momentum tail $\propto 1 / Q^{4}$. On the other hand, this solution would exponentially blow up at large values of $z$, but this is not a problem since, as previously argued, the radial dimension must be anyway supplemented with a cutoff at $z=1 / \Lambda$. From the perspective of the boundary gauge theory, this scale $\Lambda$ plays several roles, so like in QCD: it acts

with the radial distance denoted as $z$. Thus by 'large radial distances' we mean large separations from the Minkowski boundary of $\mathrm{AdS}_{5}$, which in these coordinates lies at $z=0$. 
as an infrared cutoff for quantum fluctuations, it sets the scale for energy-momentum density in the 'nucleus', and also the characteristic scale for transverse inhomogeneity.

The introduction of this radial cutoff is clearly an ad hoc procedure (it spoils the exactness of the solution) and any quantity which is sensitive to the details of this procedure is modeldependent. This is probably the case for the collision between two such shockwaves, but this is hardly a surprise: already in QCD, total hadronic cross-sections are dominated by soft interactions and thus are sensitive to the physics of confinement. But even in that case, one may hope that the thermalization process in the late stages of the collision is less sensitive to the details of the cutoff. On the other hand, the collision between this 'nucleus' and a 'hard' probe with high transverse resolution $Q \gg \Lambda$ is probing the shockwave metric only at small radial distances $z \lesssim 1 / Q \ll 1 / \Lambda$, and hence it is completely insensitive to the infrared cutoff. Once again, this is similar to QCD where deep inelastic scattering at high- $Q^{2}$ is insensitive to confinement.

The above considerations are illustrated by our calculations of deep inelastic scattering (DIS) off the shockwave, which represent most of the material in this paper. The first calculations of DIS at strong coupling within the gauge/string duality referred to some other types of targets: a 'glueball' bound state $[33,36-38]$ (whose gravity dual is a normalizable dilaton state in $\mathrm{AdS}_{5}$ ) and a finite temperature plasma (as represented by the $\mathrm{AdS}_{5}$ black-brane) [34,35]. More recently, Refs. [39,40] presented the corresponding calculations for a shockwave target (a 'nucleus', or a 'plasma slice'), but their respective results appear to disagree with each other. The main difference refers to the energy dependence of the saturation momentum $Q_{s}$, which is the characteristic scale for the onset of unitarity corrections: for $Q \gg Q_{s}$, the scattering is weak, whereas for $Q \lesssim Q_{s}$ it reaches the unitarity bound, or 'black disk', limit.

Already at weak coupling, $Q_{s}$ is known to grow quite fast with the energy, as an inverse power of the Bjorken $x$ variable, due to the rapid increase in the gluon density at small $x$ via bremsstrahlung [6]. At strong coupling, one expects parton branching to be faster and to 'quasidemocratically' divide the energy among the daughter partons [33,34,36, 41-43]. Accordingly, all partons should fragment down to very small values of $x$ and then the saturation momentum should grow with $1 / x$ even faster. This is indeed what one found in Ref. [34,36,40] for various types of hadronic targets: dilaton, infinite plasma, and a plasma slice. Moreover, the energy dependence emerging from these calculations looks very natural from the viewpoint of supergravity: the scattering proceeds via multiple graviton exchanges, so the saturation momentum inherits the energy dependence of the graviton propagator, which implies $Q_{s}^{2} \propto 1 / x$. This specific energy dependence is also necessary to ensure energy-momentum conservation, as we shall later explain. On the other hand, Ref. [39] reported a rather counterintuitive result for $Q_{s}$, which becomes independent of $x$ in the high energy limit. Although that analysis uses a different projectile - namely, a small 'color dipole' (dual to a Nambu-Goto open string in $\mathrm{AdS}_{5}$ ) instead of a virtual photon - , it is unlikely that this can explain the dramatically different result obtained there for the saturation momentum ${ }^{3}$. Indeed, the latter is an intrinsic property

\footnotetext{
${ }^{2}$ The picture is more subtle in the case of an infinite plasma, and the associated saturation scale shows a faster rise with the energy, namely $Q_{s}^{2} \propto 1 / x^{2}$, but the additional power of $1 / x$ is understood simply as the coherence length of the virtual photon $[34,35]$.

${ }^{3}$ In fact, we shall find that the supergravity field dual to the virtual photon describes a dipolar partonic fluctuation of the latter, so the DIS proceeds via the dipole scattering off the shockwave, so like in QCD at weak coupling. Hence even the physical setup looks a priori similar in Refs. [34,36,40] and respectively [39].
} 
of the target, hence it must be insensitive to the precise nature of the projectile.

Our analysis in Sects. 3 and 4 will extend the previous analysis [40] of a finite--width plasma, with results which agree with Ref. [40] whenever a direct comparison is possible. Our generalization will refer to several aspects:

(i) We shall consider two types of external probes: an $\mathcal{R}$-current (so like in Ref. [40]) in Sect. 4, and a 'dilaton' in Sect. 3. The $\mathcal{R}$-current is an analog of the electromagnetic current for the $\mathcal{N}=4$ SYM theory, and is dual to an Abelian vector field propagating in the asymptotically $\mathrm{AdS}_{5}$ space-time. In the supergravity approximation, DIS amounts to solving the Maxwell equations for this vector field in the background of the $\mathrm{AdS}_{5}$ shockwave geometry ${ }^{4}$. The 'dilaton' is a supergravity scalar field which is dual to the Lagrangian density $\mathcal{L}=(1 / 4) F_{\mu \nu}^{a} F_{a}^{\mu \nu}+\ldots$ The dilaton case will be presented first, and in more detail, since the corresponding equations of motion - the Klein-Gordon equations in the shockwave geometry — turn out to be simpler.

(ii) We shall for the first time consider the impact parameter dependence of the scattering amplitude and of the saturation momentum, and in particular study the expansion of the black disk with increasing energy and the emergence of the Froissart bound. We shall perform this analysis for two types of shockwaves: that introduced in Ref. [14] and which involves a pointlike source in the bulk, and the one that we shall propose in Sect. 2 below and which allows for an arbitrary inhomogeneity in the transverse plane.

(iii) Both for the dilaton and for the $\mathcal{R}$-current, we shall construct the exact supergravity solution in great detail, by resumming multiple scattering to all orders and showing that this leads to the eikonalization of the single graviton exchange, as expected for the scattering off a shockwave. (The eikonalization was also advocated in Ref. [40], but without an explicit construction.) This requires the respective 'bulk-to-bulk' propagators in $\mathrm{AdS}_{5}$ in real time and light-cone coordinates, that will be constructed in Appendix B. Similar results in the AdS/CFT context have been previously obtained in Refs. [44-46].

Let us now summarize the main results and conclusions which will emerge from our analysis:

The DIS structure functions in the supergravity approximation come out in a factorized structure which is reminiscent of the $k_{\perp}$-factorization in QCD at weak coupling [6], with the radial distance $z$ in $\mathrm{AdS}_{5}$ playing the role of the transverse size $r_{\perp} \sim 1 / k_{\perp}$ of the partonic fluctuations of the projectile ('virtual photon') in the boundary gauge theory (as expected from the UV/IR correspondence $[26,34,35]$ ). Namely, they involve a convolution in $z$ over the square of the incoming field (the supergravity solution for the dilaton or the Maxwell field in the absence of the shockwave) times the cross-section for the scattering between this field and the shockwave at a given value of $z$. In turn, this cross-section is expressed in the eikonal approximation, as the integral over all the impact parameters of a scattering amplitude which resums multiple graviton exchanges to all orders.

For sufficiently large $Q^{2}$ and/or large values of Bjorken $x$, the multiple scattering series can be expanded out, and then the dominant contribution to the structure functions comes from the double graviton exchange (the single graviton exchange being purely real). This leading contribution is of higher twist order, meaning that there are no point-like constituents in the target, in agreement with previous analyses at strong coupling [33-38], but in sharp contrast

\footnotetext{
${ }^{4}$ For the supergravity approximation to apply to DIS, the total COM energy squared $s$ must be limited in such a way not to allow for the excitation of massive string states. The respective condition can be written as $1 / x \ll \sqrt{\lambda}[33]$, where $x \simeq Q^{2} / s$ is the Bjorken variable.
} 
with the situation at weak coupling, where the dominant contribution at large $Q^{2}$ is of twist-two order and describes parton (in QCD, quark) distributions [6, 47].

For sufficiently low $Q^{2}$ at a given value of $x$, the amplitude reaches the unitarity limit and the structure functions are large. Remarkably, at least for the $\mathcal{R}$-current, the structure functions at low $Q^{2}$ have the same parametric form as for a proton in QCD at weak coupling. This similarity suggests a physical interpretation in terms of parton saturation at strong coupling $[34,36]$ : the low $-k_{\perp}$ and low $-x$ region of the phase-space is filled with partons, with occupation numbers of order one. Note that, in pQCD, the occupation numbers at saturation are of order one only for quarks, but they are of order $1 / \lambda$ (with $\lambda=g^{2} N_{c} \ll 1$ at weak coupling) for gluons [6]. In that case, saturation is driven by gluon dynamics, namely by the enhanced radiation of gluons with small values of $x$ and their mutual interactions (quark saturate only due to their coupling to gluons). By contrast, at strong coupling, saturation is driven by quasi-democratic branching and the occupation numbers at saturation are of order one for all types of partons $[34,36]$.

The borderline between the weak-scattering regime at high- $Q^{2}$ and large $-x$, where there are no partons, and the saturation region at low $-Q^{2}$ and low $-x$ defines the saturation line, which can be expressed either as $Q^{2}=Q_{s}^{2}(x)$, or $x=x_{s}(Q)$. We find that this saturation line is exactly the same for the dilaton and the $\mathcal{R}$-current, which confirms that this is an intrinsic property of the 'nuclear' target. Specifically, we find $Q_{s}^{2}(x) \sim \Lambda^{3} L / x$, in agreement with Ref. [40]; here, $\Lambda$ is the 'confinement' scale, as introduced by the energy density in the target ${ }^{5}$, and $L$ is the longitudinal extent of the target in its rest frame. This peculiar $1 / x$-dependence of $Q_{s}$, which reflects the energy-dependence of the single graviton exchange, can be also understood via an independent argument, that we now explain.

Namely, energy-momentum conservation implies that the integral $\int_{0}^{1} \mathrm{~d} x F_{2}\left(x, Q^{2}\right)$ of the structure function $F_{2}$ has a finite limit, of order $N_{c}^{2}$, as $Q^{2} \rightarrow \infty$. At large $x \gg x_{s}(Q)$, $F_{2}\left(x, Q^{2}\right)$ has only higher-twist contributions which rapidly die away with increasing $Q^{2}$ (see Eq. (4.41)). At $x \lesssim x_{s}(Q), F_{2}\left(x, Q^{2}\right)$ is independent of $x$, due to saturation, and of order $N_{c}^{2} Q^{2}$ (see Eq. (4.42)). Hence, the integral is dominated by $x \sim x_{s}(Q)$ - the highest value of $x$ at which the structure function is still finite at large $Q^{2}-$, and can be estimated as

$$
\int_{0}^{1} \mathrm{~d} x F_{2}\left(x, Q^{2}\right) \sim x_{s} F_{2}\left(x_{s}, Q^{2}\right) \sim x_{s} N_{c}^{2} Q^{2}
$$

For this to be independent of $Q^{2}, x_{s}$ must scale as $x_{s}(Q) \sim 1 / Q^{2}$, or $Q_{s}^{2}(x) \sim 1 / x$, as announced.

So far, we did not mention the dependence of the various results, so like $Q_{s}$, upon the impact parameter $b_{\perp}$. This will be discussed at length in the main text, and the main conclusion is that, at strong coupling, the function $Q_{s}^{2}\left(x, b_{\perp}\right)$ has the same $b_{\perp}$-dependence as the energymomentum distribution in the shockwave. In particular, for a distribution which exhibits an exponential tail at large $b_{\perp}$ (as expected in the presence of confinement), $Q_{s}^{2}\left(x, b_{\perp}\right)$ has an exponential tail as well, and the black disk area grows like $\ln ^{2}(1 / x)$, that is, it saturates the Froissart bound. On the other hand, for the shockwave metric in Ref. [14], $Q_{s}^{2}\left(x, b_{\perp}\right) \sim 1 / b_{\perp}^{6}$ at large $b_{\perp}$, and the area of the black disk grows like a power of the energy, in violation of the Froissart bound.

\footnotetext{
${ }^{5}$ This is also the scale which fixes the radial cutoff at $z \sim 1 / \Lambda$ in $\operatorname{AdS}_{5}$, as previously explained, but this cutoff plays no role for DIS at $Q^{2} \gg \Lambda^{2}$.
} 


\section{Shockwaves with impact parameter dependence in $\mathbf{A d S}_{5}$}

In this section, we shall discuss various proposals for asymptotically $\mathrm{AdS}_{5}$ shockwave metrics which are intended to represent the gravity duals of a fast moving 'nucleus' (more properly, a slice of the plasma) with a non-trivial profile in impact parameter space (the two dimensional space transverse to the direction of motion). We shall work in the nucleus infinite momentum frame, that is, we shall take the nucleus to move in the positive $x^{3}$ direction with a Lorentz $\gamma$ factor which is arbitrarily large. It is then convenient to introduce light-cone coordinates,

$$
x^{+} \equiv \frac{1}{\sqrt{2}}\left(x^{0}+x^{3}\right), \quad x^{-} \equiv \frac{1}{\sqrt{2}}\left(x^{0}-x^{3}\right),
$$

in terms of which the nucleus moves in the positive $x^{+}$direction, and the only non-trivial component of its average energy-momentum tensor $\left\langle T_{\mu \nu}\right\rangle$ is the $(-,-)$ component (as this is the only one to be enhanced by the large factor $\gamma^{2}$ ). By energy-momentum conservation, $\partial^{\mu}\left\langle T_{\mu \nu}\right\rangle=0$, this component ${ }^{6} T_{--} \equiv\left\langle T_{--}\right\rangle$is independent of the light-cone time $x^{+}$. For the time being, we shall allow $T_{--}\left(x^{-}, x_{\perp}\right)$ to be an arbitrary function of $x^{-}$and the transverse coordinates $x_{\perp}=\left(x^{1}, x^{2}\right)$ (the 'impact parameter'). In practice we shall be mostly interested in the situation where the nucleus is strongly Lorentz contracted: $T_{--} \propto \delta\left(x^{-}\right)$(the 'shockwave').

We shall assume $T_{--}$to be proportional to $N_{c}^{2}$. This is unrealistic from the point of view of a real QCD problem, where a nucleus is built with colorless hadrons (protons and nucleons). But within the present AdS/CFT context, such an assumption is necessary in order to be able to study the high-energy dynamics in the large $-N_{c}$ limit, to which we would like restrict ourselves in what follows. Indeed, it is only when $T_{--} \propto N_{c}^{2}$ that the metric perturbation induced by the 'nucleus' in the 'bulk' metric of $\mathrm{AdS}_{5}$ is an effect of $\mathcal{O}(1)$ which survives when $N_{c} \rightarrow \infty$. Then, the nucleus and its interactions can be simply described in the supergravity approximation, in which one first solves the Einstein equations to determine the asymptotically $\mathrm{AdS}_{5}$ metric dual to the nucleus and then study the propagation of various projectiles in this metric.

Our subsequent construction may be viewed as a model for the gravity dual of a slice of a $\mathcal{N}=4 \mathrm{SYM}$ plasma at finite temperature $T$, which indeed has $N_{c}^{2}$ degrees of freedom per unit volume, and hence an energy density $T_{00} \sim N_{c}^{2} T^{4}$ in the plasma rest frame, yielding $T_{--} \sim \gamma^{2} N_{c}^{2} T^{4}$ in the infinite momentum frame. In theoretical studies, the plasma is generally assumed to be infinite, but this is unrealistic from a phenomenological viewpoint and, besides, the concept of plasma makes sense also for a finite volume system (at least over a finite time interval), so long as the size $L$ of the system along any direction obeys $L \gg 1 / T$. An explicit connection between the shockwave metric that we shall construct in this section and the boosted slice of the plasma will be established in Appendix A.

As mentioned in the Introduction, there are two types of proposals in the literature for shockwave metrics in $\mathrm{AdS}_{5}$ : one which introduces a gravity source in the bulk of $\mathrm{AdS}_{5}$ [14-17], and one which does not $[12,30]$ (but boundary sources are in principle allowed in the second case; see below). Both cases can be encoded in the following Einstein equations

$$
\mathcal{R}_{m n}-\frac{\mathcal{R}}{2} g_{m n}+\Lambda g_{m n}=8 \pi G_{5} J_{m n},
$$

\footnotetext{
${ }^{6}$ To avoid cumbersome notations, we shall omit the brackets denoting expectation values whenever there is no risk of confusion.
} 
where $m, n$ are 5-dimensional space-time indices, $\Lambda=-6 / R^{2}$ (with $R$ the curvature radius of $\left.\mathrm{AdS}_{5}\right)$ is the cosmological constant, $\mathcal{R} \equiv g^{m n} \mathcal{R}_{m n}=-20 / R^{2}, G_{5}$ is the Newton constant in $D=5$, and $J_{m n}$ is the stress tensor of the source localized in the bulk, for which we shall consider the two scenarios alluded to above:

1. Type-I metric: $J_{m n}=0$. This is the case considered in Refs. $[12,30]$.

2. Type-II metric: $J_{m n}$ corresponds to an ensemble of $N_{c}^{2}$ point-like 'particles' moving together within $\mathrm{AdS}_{5}$ along a null geodesic parallel to the Minkowski boundary (see Eq. (2.18) for an explicit expression). This is the case considered in Refs. [14-17].

As shown in the literature, the corresponding shockwave solutions to Eq. (2.2) can be obtained with the following Ansatz (in the so-called Fefferman-Graham coordinates)

$$
\mathrm{d} s^{2}=\frac{R^{2}}{z^{2}}\left[\mathrm{~d} z^{2}-2 \mathrm{~d} x^{+} \mathrm{d} x^{-}+\mathrm{d} x_{\perp}^{2}+h\left(z, x^{-}, x_{\perp}\right)\left(\mathrm{d} x^{-}\right)^{2}\right],
$$

for both cases: with or without bulk sources. Still in both cases, the function $h\left(z, x^{-}, x_{\perp}\right)$ is determined by the following, linear, equation

$$
\left(\partial_{z}^{2}-\frac{3}{z} \partial_{z}+\nabla_{\perp}^{2}\right) h\left(z, x^{-}, x_{\perp}\right)=-16 \pi G_{5} J_{--},
$$

which is the $(-,-)$ component of Eq. (2.2). Note that there is no 'small perturbation' assumption involved here: Eq. (2.4) is the exact consequence of the Einstein equations (2.2) for the Ansatz (2.3) and the specific stress-tensor $J_{m n}$ under consideration. Accordingly, the metric 'perturbation' $g_{--}=\left(R^{2} / z^{2}\right) h$, which describes the shockwave, needs not be parametrically small. In fact, the normalization of this perturbation is related to that of the energy-momentum tensor on the boundary, via holographic renormalization $[48,49]$. Specifically, if $h^{(4)}\left(x^{-}, x_{\perp}\right)$ is the coefficient of the $z^{4}$ term in the near-boundary $(z \rightarrow 0)$ expansion of $h\left(z, x^{-}, x_{\perp}\right)$, then

$$
T_{--}\left(x^{-}, x_{\perp}\right)=\frac{R^{3}}{4 \pi G_{5}} h^{(4)}\left(x^{-}, x_{\perp}\right)=\frac{N_{c}^{2}}{2 \pi^{2}} h^{(4)}\left(x^{-}, x_{\perp}\right),
$$

where the second equality follows after using the AdS/CFT correspondence to identify $G_{5}=$ $\pi R^{3} / 2 N_{c}^{2}$. As anticipated, $T_{--}$must scale like $N_{c}^{2}$ for the metric perturbation not to be parametrically small.

We shall now successively consider the two interesting cases. In this process, we shall recall some results from the literature, and we shall correct and extend the proposal in Refs. [12,30].

\subsection{Type-I metric: no source in the bulk}

In this case we need the non-trivial solutions to the homogeneous version of Eq. (2.4). Consider first the case where there is no dependence upon $x_{\perp}$, corresponding to a nucleus which is uniform in impact parameter space (an infinite, uniform, wall). Then the solution reads

$$
h\left(z, x^{-}\right)=\frac{2 \pi^{2}}{N_{c}^{2}} z^{4} T_{--}\left(x^{-}\right)
$$

where the normalization has been fixed according to Eq. (2.5). With this expression for $h$, the metric (2.3) is the original shockwave metric proposed by Janik and Peschanski [12]. Although 
an exact solution to the (homogeneous) Einstein equations, this metric has nevertheless the drawback to have a singular point at $z \rightarrow \infty$. We shall return to this issue later on.

Consider now the homogeneous version of Eq. (2.4) corresponding to a generic profile in $x_{\perp}$. It is then convenient to perform a Fourier transform to transverse momentum space, which yields the following equation

$$
\left(\partial_{z}^{2}-\frac{3}{z} \partial_{z}-k_{\perp}^{2}\right) h\left(z, x^{-}, k_{\perp}\right)=0 .
$$

The general solution is expressed in terms of modified Bessel functions of second rank:

$$
h\left(z, x^{-}, k_{\perp}\right)=\frac{1}{2}\left(z k_{\perp}\right)^{2}\left[c_{1}\left(x^{-}, k_{\perp}\right) \mathrm{K}_{2}\left(z k_{\perp}\right)+c_{2}\left(x^{-}, k_{\perp}\right) \mathrm{I}_{2}\left(z k_{\perp}\right)\right] .
$$

The near-boundary expansion of this solution reads as follows (up to order $z^{4}$ )

$$
\begin{aligned}
h\left(z, x^{-}, k_{\perp}\right)= & c_{1}\left(x^{-}, k_{\perp}\right)\left[1-\frac{z^{2} k_{\perp}^{2}}{4}+\frac{z^{4} k_{\perp}^{4}}{32}\left(-2 \ln \left(z k_{\perp} / 2\right)+3 / 2-2 \gamma_{E}\right)\right]+ \\
& +c_{2}\left(x^{-}, k_{\perp}\right) \frac{z^{4} k_{\perp}^{4}}{16}+\cdots,
\end{aligned}
$$

that is, the component proportional to $\mathrm{I}_{2}\left(z k_{\perp}\right)$ vanishes like $z^{4}$ when $z \rightarrow 0$, while that proportional to $\mathrm{K}_{2}\left(z k_{\perp}\right)$ approaches a non-zero value in this limit. Consider also the large $z$ behavior: for $z k_{\perp} \gg 1, \mathrm{~K}_{2}\left(z k_{\perp}\right)$ is exponentially decreasing, while $\mathrm{I}_{2}\left(z k_{\perp}\right)$ is exponentially increasing. For what follows, it is interesting to keep in mind that the homogeneous (in the sense of no dependence upon $x_{\perp}$ ) solution in Eq. (2.6) corresponds to the limit $k_{\perp} \rightarrow 0$ of the $\mathrm{I}_{2}$-piece of the general solution Eq. (2.8).

In the framework of AdS/CFT, the solutions which diverge as $z \rightarrow \infty$ are unacceptable and must be discarded. In view of that, it might look natural to enforce $c_{2}=0$ in Eq. (2.8): this is the common strategy for computing correlation functions in the vacuum of $\mathcal{N}=4$ SYM (here, the correlators of $T_{\mu \nu}$ ) [7-9], and this was also the proposal made in Ref. [30] for constructing the gravity dual of a nucleus. However, in what follows we shall argue that choosing $c_{2}=0$ in Eq. (2.8) leads to a physically unacceptable picture for a nucleus. (In particular, this would also exclude the homogeneous shockwave (2.6), which as alluded to above represents the limit $k_{\perp} \rightarrow 0$ of the piece of the solution proportional to $c_{2}$.) A more sensible choice, which is physically motivated, is to take $c_{1}=0$ and introduce a cutoff in the radial direction of $\mathrm{AdS}_{5}$ at a distance $z_{\Lambda}=1 / \Lambda$, with $\Lambda$ playing the role of an infrared cutoff in the boundary gauge theory. To motivate this proposal, let us first explain the difficulties with the original choice in Ref. [30].

As just mentioned, taking $c_{2}=0$ is the standard choice for computing the correlators of $T_{\mu \nu}$ in $\mathcal{N}=4 \mathrm{SYM}$. In that case, the metric perturbation has a non-zero limit on the boundary,

$$
h_{\mu \nu}\left(x^{-}, k_{\perp}\right)=\lim _{z \rightarrow 0}\left[\frac{z^{2}}{R^{2}} g_{\mu \nu}\left(z, x^{-}, k_{\perp}\right)\right]-\eta_{\mu \nu}=\delta_{\mu-} \delta_{\nu-} c_{1}\left(x^{-}, k_{\perp}\right),
$$

which acts as an external source (not to be confused with the bulk source $J_{m n}$ in Eq. (2.2)), which couples to $T_{\mu \nu}$ in the boundary gauge theory. This external source induces a non-vanishing expectation value for $T_{\mu \nu}$, which is proportional to the source and can be read off Eq. (2.5) :

$$
\left\langle T_{\mu \nu}\left(x^{-}, k_{\perp}\right)\right\rangle=\delta_{\mu-} \delta_{\nu-} \frac{N_{c}^{2}}{64 \pi^{2}} k_{\perp}^{4} \ln \left(\frac{\mu^{2}}{k_{\perp}^{2}}\right) c_{1}\left(x^{-}, k_{\perp}\right) .
$$


Here, $\mu$ is scale for ultraviolet renormalization in the gauge theory, as introduced by the removal of the logarithmic singularity at $z \rightarrow 0$ manifest in Eq. (2.9). (The finite terms beyond the logarithm which are also visible in Eq. (2.9) have been absorbed in the definition of $\mu$.) By taking a functional derivative in Eq. (2.11) with respect to $h_{--}=c_{1}$, one obtains the only non-vanishing 2-point function of $T_{\mu \nu}$ within the present kinematics (in momentum space and with trivial delta functions removed) :

$$
\left\langle T_{--}\left(k_{\perp}\right) T_{++}\left(-k_{\perp}\right)\right\rangle=\frac{N_{c}^{2}}{64 \pi^{2}} k_{\perp}^{4} \ln \left(\frac{\mu^{2}}{k_{\perp}^{2}}\right) .
$$

This is indeed the expected result ${ }^{7}$, with the specific $k_{\perp}$-dependence in the r.h.s. reflecting the conformal symmetry of $\mathcal{N}=4$ SYM (see, e.g., the discussion in [8]).

Within this standard procedure, the external source $h_{\mu \nu}$ plays no dynamical role, but rather acts as a device for generating vacuum correlations via functional differentiation. By contrast, in Ref. [30] it has been proposed to use a similar procedure with a suitable choice for the function $c_{1}\left(x^{-}, k_{\perp}\right)$ in such a way to generate, via Eq. (2.11), an energy-momentum profile which physically would correspond to an ultrarelativistic nucleus. However, Ref. [30] showed no explicit proposal for such a function $c_{1}\left(x^{-}, k_{\perp}\right)$, and in fact we shall now argue that there is no meaningful solution of this type.

Our main objection to the proposal in Ref. [30] is of physical nature, but it also gets reflected in severe technical difficulties, which lead to paradoxes. Let us start with one of these paradoxes, before we explain the deeper origin of the problem. Returning to the simpler notation $T_{--} \equiv\left\langle T_{--}\right\rangle$, we notice that one must have $T_{--}\left(k_{\perp}=0\right)>0$ because

$$
T_{--}\left(x^{-}, k_{\perp}=0\right)=\int \mathrm{d}^{2} x_{\perp} T_{--}\left(x^{-}, x_{\perp}\right)
$$

where $T_{--}\left(x^{-}, x_{\perp}\right)$ is the positive semidefinite energy density in the transverse plane. For this to be consistent with Eq. (2.11) one should have $c_{1}$ behaving like $1 / k_{\perp}^{4}$ when $k_{\perp} \rightarrow 0$. But then the Fourier transform of $h\left(z, x^{-}, k_{\perp}\right)$ back to the transverse coordinate space is ill defined, because of an infrared divergence at $k_{\perp} \rightarrow 0$. On the other hand, there is clearly no similar problem if one chooses $c_{1}=0$ and $c_{2} \neq 0$ in Eq. (2.8), since then one can take $c_{2} \sim 1 / k_{\perp}^{4}$ as $k_{\perp} \rightarrow 0$ without generating infrared problems, as obvious from the expansion (2.9).

That this is the only meaningful choice, it is also suggested by the behavior (2.9) of the metric near $z=0$ together with its physical interpretation according to the AdS/CFT dictionary. Via the UV/IR correspondence [26,31-35], the inverse $1 / z$ of the radial distance in $\mathrm{AdS}_{5}$ is mapped onto the transverse momenta (or virtualities) of the quantum fluctuations in the boundary gauge theory. Hence an expression like Eq. (2.8) for the metric perturbation should be viewed as encoding information about two types of transverse momenta: the momentum $k_{\perp}$ which via the Fourier mode $c_{2}\left(x^{-}, k_{\perp}\right)$ determines the profile of the nucleus in impact parameter space, and the momentum $p_{\perp} \sim 1 / z$ which refers to the quantum modes that we would like to include in our description of the nucleus and of its high-energy interactions.

\footnotetext{
${ }^{7}$ In the corresponding expressions in the literature, $k_{\perp}^{2}$ is generally replaced by the invariant $4-$ momentum squared $k^{2}=k_{\perp}^{2}-2 k^{+} k^{-}$. Note however that for the present set-up we have $k^{-}=0$ since there is no dependence upon $x^{+}$.
} 
On physical grounds, in particular in view of our experience with QCD, we expect the bulk of the modes in a hadron wavefunction to be concentrated at 'soft' momenta, of the order of some infrared cutoff $\Lambda$, whereas at much larger momenta $p_{\perp} \gg \Lambda$ the distribution should rapidly decrease, typically according to a power law $\left(1 / p_{\perp}\right)^{\Delta}$. The exponent $\Delta$ is equal to 2 for the partonic tail produced via bremsstrahlung in QCD at weak coupling, but it is equal to 4 or larger in all the situations where a gravity dual has been identified for a hadronic system at strong coupling. For instance, $\Delta=4$ in the case of the $\mathrm{AdS}_{5}$ black-brane geometry dual to the $\mathcal{N}=4$ SYM plasma [11], and also for the uniform shockwave metric in Eq. (2.6]) [12], or for the metric induced by a pointlike source in the bulk of $\mathrm{AdS}_{5}$ [17] (see Eq. (2.22) below). Furthermore, for a normalizable dilaton state in $\mathrm{AdS}_{5}[8,9]$, which is dual to a 'glueball', one has $\Delta=2+\sqrt{m^{2} R^{2}+4} \geq 4$, where the dilaton mass $m$ is proportional to the scale $\Lambda$ which enters via the radial cutoff at $z \sim 1 / \Lambda$ [23]. Such a large value for $\Delta$ at strong coupling can be understood as the absence of partons with high momenta [33-36], an interpretation to which we shall return in Sects. 3 and 4. Clearly, $\Delta=4$ is also the prediction of Eq. (2.8) provided one takes $c_{1}=0$ (cf. Eq. (2.9) ). On the other hand, with $c_{2}=0$, Eq. (2.8) predicts a dominant behavior near $z=0$ which is independent of $z$, that is, a flat momentum spectrum $(\Delta=0)$ in the high momentum regime, which physically makes no sense.

The previous examples also show that the behavior in $z^{4}$ of the metric perturbation holds only for sufficiently small values of $z$, corresponding to the ultraviolet behavior of the dual gauge theory. But this growth is cut off at some larger value of $z$, whose inverse plays the role of an infrared cutoff in the gauge theory. Such a cutoff is necessary to introduce the analog of matter (plasma or hadronic bound state) in the otherwise conformal SYM field theory. For instance, this scale is provided by the black hole horizon at $z \sim 1 / T$ for the $\operatorname{AdS}_{5}$-Schwarzschild metric, by the radial position of the source in the bulk for the shockwave metric in Ref. [17] (see Sect. 2.2 below), and by an explicit cutoff at large values of $z$ in the construction of the glueball bound state $[22,23,25]$.

At a first sight, the uniform shockwave metric (2.6) seems not to involve any such a scale, but this is only illusory: written as it stands, the metric perturbation in Eq. (2.6) blows up at $z \rightarrow \infty$ and this divergence is a genuine space-time singularity, and not just a coordinate singularity. As mentioned in the Introduction, this singularity is quite elusive, as it does not show up in any of the curvature scalars computed from this metric. Rather, it can be identified via a more general criterion for space-time singularities, known as $g$-incompleteness (with $g$ standing for "geodesic") [29]. Namely, in an acceptable space-time, which is $g$-complete, any time-like or null-like geodesic can be extended up to arbitrary values in their affine parameters (like the proper time). The contrary would imply the existence of observers whose history ends or begin at a finite proper time. On the other hand, for the Janik-Peschanski metric one can construct time-like geodesics which, starting at a generic point $z_{0}$, reach infinity after a finite value of the proper time. (See [27] for an analogous construction in the Kaigodorov spacetime, which is the 4-dimensional version of the Janik-Peschanski metric.) Hence, this metric is $g$-incomplete, and thus unacceptable. One can effectively 'hide' the space-time singularity at $z \rightarrow \infty$ by introducing a radial cutoff at $z=1 / \Lambda$, which for that purpose plays the same role as the black hole horizon within the $\mathrm{AdS}_{5}$ black-brane metric. But once this is done, it is no more disturbing to use a general metric perturbation $\propto z^{2} \mathrm{I}_{2}\left(z k_{\perp}\right)$, which has the right behavior 
at small $z$ and allows for a generic transverse inhomogeneity, instead of the uniform shockwave in Eq. (2.6).

To summarize, our proposal for a gravity dual to a large nucleus is given by the asymptotically $\mathrm{AdS}_{5}$ metric in Eq. (2.3) which applies for $z \leq z_{\Lambda} \equiv 1 / \Lambda$ and where the function $h$ is given, in transverse momentum space, by Eq. (2.8) with $c_{1}=0$ and $c_{2}$ related to the energy-momentum tensor of the nucleus that we would like to describe via

$$
T_{--}\left(x^{-}, k_{\perp}\right)=\frac{N_{c}^{2}}{32 \pi^{2}} k_{\perp}^{4} c_{2}\left(x^{-}, k_{\perp}\right) .
$$

The example of the 'plasma slice' previously discussed suggests that a physically reasonable choice for $T_{--}$would be

$$
T_{--}\left(x^{-}, x_{\perp}\right)=\gamma^{2} N_{c}^{2} \Lambda^{4} f\left(x^{-}, x_{\perp} \Lambda\right),
$$

where the dimensionless function $f$ describes the shape of the 'nucleus' in longitudinal and transverse directions. The transverse inhomogeneity is controlled by the 'soft' scale $\Lambda$, so like in QCD. The longitudinal support is concentrated at $\left|x^{-}\right| \lesssim L / \gamma$ with $L$ the width of the 'nucleus' in its rest frame. In the high energy limit, we can replace this by a $\delta$-function in $x^{-}$:

$$
f\left(x^{-}, x_{\perp} \Lambda\right)=f\left(x_{\perp} \Lambda\right) \frac{L}{\gamma} \delta\left(x^{-}\right), \quad \Lambda^{2} \int \mathrm{d}^{2} x_{\perp} f\left(x_{\perp} \Lambda\right)=1,
$$

where the normalization of the new function $f\left(x_{\perp} \Lambda\right)$ has been chosen for convenience. Some reasonable choices for $f$, inspired by our experience with QCD, would be a Gaussian, or a Woods-Saxon profile which falls exponentially for distances far away from the center, that is for $x_{\perp} \Lambda \gg 1$. These choices lead to the following model for the metric perturbation:

$$
h\left(z, x^{-}, x_{\perp}\right)=16 \pi^{2} \gamma L \Lambda^{2} z^{2} \delta\left(x^{-}\right) \int \frac{\mathrm{d}^{2} k_{\perp}}{(2 \pi)^{2} k_{\perp}^{2}} \mathrm{e}^{i k_{\perp} x_{\perp}} \tilde{f}\left(k_{\perp} / \Lambda\right) \mathrm{I}_{2}\left(z k_{\perp}\right)
$$

where $\tilde{f}\left(k_{\perp} / \Lambda\right)$ is the Fourier transform of $\Lambda^{2} f\left(x_{\perp} \Lambda\right)$ (so it is dimensionless), and it has support at $k_{\perp} \lesssim \Lambda$. A radial cutoff at $z \sim z_{\Lambda}$ is implicit. Then, clearly, the argument of $\mathrm{I}_{2}$ can never become large.

Our prescription for cutting off $\mathrm{AdS}$ at $z=z_{\Lambda}$ is merely a model, and any calculation which is sensitive to large values of $z$ (so like the scattering between two shockwaves) will be strongly sensitive to the details of this model - the value of $\Lambda$ and the specific procedure used for its implementation. Fortunately, there are also interesting phenomena, so like the deep inelastic scattering to be considered in Sects. 3 and 4 , which are controlled by the 'hard' $\left(p_{\perp} \gg \Lambda\right)$ part of the spectrum - in the AdS framework, by the behavior of the metric near the boundary at $z=0$ - and thus are completely insensitive to the model used to cutoff $\mathrm{AdS}_{5}$ at large $z$.

\subsection{Type-II metric: pointlike source in the bulk}

We now briefly describe the shockwave proposal in Ref. [17], which involves a source in the bulk. We shall take this source to be composed of $N_{c}^{2}$ point-like 'particles' moving together ${ }^{8}$ along

\footnotetext{
${ }^{8}$ Ref. [17] mentioned only a single such a particle, but here we shall consider a collection of $N_{c}^{2}$ of them, in order to achieve a metric perturbation of order one. The alternative possibility, which would be to take a single particle but with ultrahigh energy $p^{+} \sim N_{c}^{2}$, would be inconsistent with the use of the supergravity approximation for any collision involving that 'particle' $[33,36]$.
} 
the trajectory defined by: $x^{-}=0, x_{\perp}=0$, and $z=z_{*}$, with $z_{*}$ a positive constant. Then the only non-zero component of $J_{m n}$ is $J_{--}$, and is given by [17]

$$
J_{--}=p^{+} N_{c}^{2} \frac{z^{3}}{R^{3}} \delta\left(x^{-}\right) \delta^{(2)}\left(x_{\perp}\right) \delta\left(z-z_{*}\right),
$$

with $p^{+}$the light-cone longitudinal momentum of a single 'particle'. The solution to Eq. (2.4) corresponding to this current reads

$$
h\left(z, x^{-}, x_{\perp}\right)=\frac{\pi p^{+}}{16} \frac{z z_{*}}{q^{3}}{ }_{2} F_{1}(3,5 / 2,5,-1 / q) \delta\left(x^{-}\right)
$$

where

$$
q \equiv \frac{x_{\perp}^{2}+\left(z-z_{*}\right)^{2}}{4 z z_{*}}
$$

is the so called 'chordal distance' ${ }^{9}$ between the point $\left(z, x_{\perp}\right)$ where we measure the metric and the position $\left(z_{*}, x_{\perp}=0\right)$ of the pointlike source in the bulk, and the hypergeometric function takes a rather simple form:

$$
{ }_{2} F_{1}(3,5 / 2,5,-1 / q)=16 q^{3} \frac{1+8 q(1+q)-4 \sqrt{q(1+q)}(1+2 q)}{\sqrt{q(1+q)}} .
$$

As anticipated, the energy $E$ of the particle must scale like $N_{c}^{2}$ in order for the metric perturbation Eq. (2.19) to be non-negligible. Note that $q \gg 1$ for both small $\left(z \ll z_{*}\right)$ and large $\left(z \gg z_{*}\right)$ values of $z$. Using ${ }_{2} F_{1} \simeq 1-3 / 2 q \approx 1$ when $q \gg 1$, we deduce

$$
h\left(z, x^{-}, x_{\perp}\right) \simeq \frac{4 \pi p^{+}\left(z z_{*}\right)^{4}}{\left(x_{\perp}^{2}+z_{*}^{2}\right)^{3}} \delta\left(x^{-}\right) \quad \text { for } \quad z \ll z_{*},
$$

and respectively

$$
h\left(z, x^{-}, x_{\perp}\right) \simeq \frac{4 \pi p^{+}\left(z z_{*}\right)^{4}}{\left(x_{\perp}^{2}+z^{2}\right)^{3}} \delta\left(x^{-}\right) \quad \text { for } \quad z \gg z_{*}
$$

so, in particular, the metric perturbation dies away as $1 / z^{2}$ when $z \rightarrow \infty$.

The associated energy-momentum tensor in the boundary gauge theory follows from Eq. (2.5):

$$
T_{--}\left(x^{-}, x_{\perp}\right)=\frac{p^{+} N_{c}^{2}}{\pi} \frac{2 z_{*}^{4}}{\left(x_{\perp}^{2}+z_{*}^{2}\right)^{3}} \delta\left(x^{-}\right) .
$$

This is essentially uniform so long as $x_{\perp} \ll z_{*}$ but it decreases like $1 / x_{\perp}^{6}$ for $x_{\perp} \gg z_{*}$. We see that $1 / z^{*}$ plays the same role as the 'soft' momentum scale $\Lambda$ introduced previously, in the sense of fixing the scale for transverse inhomogeneity in the nucleus. But unlike in that previous case, now there is no need to explicitly cut off the radial dimension of $\mathrm{AdS}_{5}$, since the metric perturbation Eq. 2.19) dies away, like $1 / z^{2}$, when $z \rightarrow \infty$.

\footnotetext{
${ }^{9}$ The chordal distance is the $S O(3,1)$-invariant distance, i.e. the analog of the radial distance, for the hyperbolic space $H_{3}$ spanned by the coordinates $\left(z, x_{\perp}\right)$.
} 


\section{DIS off the shockwave: the dilaton}

In this and the next coming section, we shall consider the supergravity problem dual to the deep inelastic scattering (DIS) between a 'hard' (i.e., highly virtual) external current and a nucleus in the $\mathcal{N}=4 \mathrm{SYM}$ theory. The 'nucleus' should be thought off as a slice of a plasma and it will be described as a shockwave, as already explained. Its construction requires an infrared cutoff $\Lambda$, but the details of this cutoff will be unimportant for the hard process at hand. The 'current' generally associated with DIS is a virtual photon with space-like virtuality which couples to the electromagnetic current of the nucleus. Within $\mathcal{N}=4 \mathrm{SYM}$, this 'electromagnetic current' can be represented by the conserved $\mathcal{R}$-current carried by (adjoint) fermionic and scalar fields in the Lagrangian. The DIS of such an $\mathcal{R}$-current will be addressed in Sect. $⿴$. But before doing that, it is preferable to introduce the formalism in the simpler context of the scalar 'current' $J=(1 / 4) F_{\mu \nu}^{a} F_{a}^{\mu \nu}$ ( $a$ is the $\mathrm{SU}\left(N_{c}\right)$ color index). This operator is interesting in itself, since it couples to the gluons inside the nuclear wavefunction, and hence is a direct probe of the gluon distribution. The 'bulk' AdS field dual to this operator is the massless dilaton field, which obeys the simplest equation of motion in supergravity: the Klein-Gordon equation in the relevant (here, the shockwave) metric. For simplicity, we shall refer to the projectile as the 'dilaton' although, strictly speaking, it is the operator $J$, and not the dilaton, which undergoes DIS in the boundary gauge theory.

\subsection{Formalism and structure function}

As before, we shall work in the infinite momentum frame of the 'target' (the nucleus), which therefore will be taken to be Lorentz contracted to a $\delta$-function at $x^{-}=0$. The 'projectile' (the dilaton) propagates in the negative $x^{3}$ direction (a 'left mover'), with space-like momentum $q^{\mu}$. In light-cone coordinates, we have $q^{-}>0$ and $q^{+}<0$, and we take $q_{\perp}=0$ for convenience; hence, the virtuality of the projectile reads $Q^{2} \equiv q^{\mu} q_{\mu}=-2 q^{+} q^{-}>0$. As usual with DIS, it is convenient to express the total cross-section (or the 'structure function') in terms of $Q^{2}$ and the Bjorken $-x$ variable, defined as

$$
x \equiv \frac{Q^{2}}{-2 q \cdot p}=\frac{Q^{2}}{2 q^{-} p^{+}}=\frac{Q^{2}}{2 q^{-} \gamma \Lambda}
$$

where $p^{\mu}=\delta^{\mu+} p^{+}$is the momentum of a typical constituent of the target: $p^{+}=\gamma \Lambda$ with $\Lambda$ the characteristic momentum scale in the target rest frame (e.g., $\Lambda=T$ for a slice of a finitetemperature plasma) and $\gamma \gg 1$ the nuclear boost factor. The kinematic conditions of interest are such that $Q^{2} \gg \Lambda^{2}$ and $x \ll 1$.

Via the optical theorem, the DIS structure function $F\left(x, Q^{2}\right)$ is expressed as the imaginary part of the forward scattering amplitude for Compton scattering:

$$
F\left(x, Q^{2}\right)=\operatorname{Im} \Pi\left(x, Q^{2}\right),
$$

with the 'polarization function' $\Pi\left(x, Q^{2}\right)$ usually written as

$$
\Pi\left(x, Q^{2}\right) \equiv i \int \mathrm{d}^{4} r \mathrm{e}^{-i q \cdot r}\langle P|\mathrm{~T}\{J(x) J(y)\}| P\rangle,
$$

where $r^{\mu}=x^{\mu}-y^{\mu}$. However, as above indicated, this writing is appropriate only when the target is in a state $|P\rangle$ with given 4 -momentum $P^{\mu}$, which is translationally-invariant. Here, however, 
the target is represented as a shockwave which is localized in space and inhomogeneous in both longitudinal $\left(x^{-}\right)$and transverse $\left(x_{\perp}\right)$ directions. Accordingly, the current-current correlator computed in this shockwave background depends not only upon the relative separation $r^{\mu}$, but also upon the central coordinates $b^{-}$and $b_{\perp}$ (defined as $b^{\mu}=\left(x^{\mu}+y^{\mu}\right) / 2$ ). To obtain a structure function which depends only upon the kinematical variables $x$ and $Q^{2}$, we shall follow the prescription in Ref. [50] and average out the central coordinates. The longitudinal extent of the target is of order $L / \gamma$ (recall that $L$ denotes its width in its rest frame) whereas the scale for inhomogeneity in the transverse plane is set by $\Lambda$. We shall thus replace Eq. (3.3) by (the precise normalization is irrelevant at this point)

$$
\Pi\left(x, Q^{2}\right)=(\gamma / L) \Lambda^{2} \int \mathrm{d}^{4} r \mathrm{~d} b^{-} \mathrm{d}^{2} b_{\perp} \mathrm{e}^{i q \cdot r} i \Theta\left(x_{0}\right)\langle[J(b+r / 2), J(b-r / 2)]\rangle,
$$

where the expectation value is now computed according to the AdS/CFT correspondence (see below) and we have replaced for convenience the time-ordered correlator by the retarded one. Both types of correlators yield the same imaginary part, but the retarded one is more easy to evaluate in the context of AdS/CFT; see e.g. [3]. Note that $J \propto\left(F_{\mu \nu}\right)^{2}$ has mass dimension 4, so $\Pi$ will have dimension 4.

Let $\Pi(x, y)$ denote the (retarded) 2-point function of $J$ which enters Eq. (3.4). According to the AdS/CFT correspondence, this is formally obtained as

$$
\Pi(x, y)=\frac{\delta S_{c l}}{\delta \phi_{b}(x) \delta \phi_{b}(y)}
$$

where $S_{c l}$ is the action of the dilaton field, that is,

$$
S=-\frac{N_{c}^{2}}{16 \pi^{2} R^{3}} \int \mathrm{d}^{4} x \mathrm{~d} z \sqrt{-g} g^{n m} \partial_{n} \phi \partial_{m} \phi,
$$

evaluated with the solution $\phi(z, x)$ to the classical equations of motion,

$$
\partial_{m}\left(\sqrt{-g} g^{m n} \partial_{n} \phi\right)=0
$$

obeying the following boundary condition at $z=0$ :

$$
\lim _{z \rightarrow 0} \phi(z, x)=\phi_{b}(x) .
$$

On physical grounds, we need the boundary field $\phi_{b}(x)$ to be the plane-wave

$$
\phi_{b}(x)=\mathrm{e}^{-i\left(q^{-} x^{+}+q^{+} x^{-}\right)} \tilde{\phi}_{b}
$$

( $\tilde{\phi}_{b}$ is simply a number), but at intermediate steps we shall consider a generic function $\phi_{b}(x)$, for the purposes of functional differentiation. Using the equations of motion, one can perform the integral over $z$ in Eq. (3.6) and obtain

$$
S_{c l}=\left.\frac{N_{c}^{2}}{16 \pi^{2}} \int \mathrm{d}^{4} x \frac{1}{z^{3}} \phi(z, x) \partial_{z} \phi(z, x)\right|_{z=0}
$$

Note that the boundary condition (3.8) is not enough to fully specify the classical solution, since Eq. (3.7) is a second order differential equation in $z$. Furthermore, the above procedure 
cannot reproduce the imaginary part of the retarded correlator, as obvious from the fact that the 2 -point function generated via Eq. (3.5) is symmetric in its arguments. We shall return to these problems later on.

Eq. (3.7) is written in the shockwave metric (2.3), which is independent of the light-cone time $x^{+}$. Thus, the equations are homogeneous in $x^{+}$, meaning that the variable $q^{-}$introduced by the projectile is conserved by the dynamics. It is therefore sufficient to restrict ourselves to the respective Fourier mode, by writing

$$
\phi\left(z, x^{+}, x^{-}, x_{\perp}\right)=\mathrm{e}^{-i q^{-} x^{+}} \phi\left(z, x^{-}, x_{\perp}\right) .
$$

(The function $\phi\left(z, x^{-}, x_{\perp}\right)$ in the r.h.s. depends upon $q^{-}$, but this dependence is kept implicit.) Then the equation of motion is more explicitly written as (with the notation $x=\left(x^{-}, x_{\perp}\right)$ )

$$
\left(\partial_{z}^{2}-\frac{3}{z} \partial_{z}+2 i q^{-} \partial_{-}+\nabla_{\perp}^{2}\right) \phi(z, x)=-\left(q^{-}\right)^{2} h(z, x) \phi(z, x) .
$$

We have separated in the right hand side the interaction piece, which describes the scattering between the dilaton and the shockwave. We shall correspondingly decompose the total field $\phi$ into its 'incoming', or 'vacuum', piece $\phi_{0}$ and the 'scattering' piece $\phi_{s}$ :

$$
\phi(z, x)=\phi_{0}(z, x)+\phi_{s}(z, x)
$$

We construct $\phi_{0}$ in such a way to satisfy the boundary condition (3.8), hence

$$
\lim _{z \rightarrow 0} \phi_{0}(x, z)=\phi_{b}(x), \quad \lim _{z \rightarrow 0} \phi_{s}(x, z)=0 .
$$

The vacuum problem is formally similar to the source-free version of Eq. (2.4) that we have already solved: the equation is homogeneous in space and time, so the solution corresponding to the boundary field (3.9) is of the form $\phi_{0}(z, x)=\mathrm{e}^{-i q^{+} x^{-}} \phi_{0}(z)$. The function $\phi_{0}(z)$ obeys the equation obtained by replacing $k_{\perp}^{2} \rightarrow-2 q^{+} q^{-}=Q^{2}>0$ in Eq. (2.7) with the boundary condition $\phi_{0}(z \rightarrow 0)=\tilde{\phi}_{b}$. Clearly the unique acceptable solution is (compare to Eq. (2.8))

$$
\phi_{0}(z)=\frac{1}{2}(Q z)^{2} \mathrm{~K}_{2}(Q z) \tilde{\phi}_{b} .
$$

This solution, together with Eqs. (3.5) and (3.10), yields the vacuum component $\Pi_{0}\left(Q^{2}\right)$ of the polarization function in a form entirely similar to Eq. (2.12) :

$$
\Pi_{0}\left(Q^{2}\right) \equiv i \int \mathrm{d}^{4} x \mathrm{e}^{-i q \cdot x}\langle 0|\mathrm{~T}\{J(x) J(0)\}| 0\rangle=\frac{N_{c}^{2} Q^{4}}{64 \pi^{2}} \ln \left(\frac{\mu^{2}}{Q^{2}}\right) .
$$

For what follows, it is useful to rewrite Eq. (3.15) in a more general way, which features the vacuum boundary-to-bulk propagator $D_{0}(z, x-y)$ :

$$
\phi_{0}(z, x)=\int \mathrm{d}^{3} y D_{0}(z, x-y) \phi_{b}(y), \quad D_{0}(z, k)=\frac{1}{2}(K z)^{2} \mathrm{~K}_{2}(K z),
$$

where the momentum-space version of $D_{0}$ was written for the space-like kinematics of interest here: $K^{2} \equiv-2 k^{+} q^{-}+k_{\perp}^{2}>0$. Via manipulations to be described in the next section, we will construct the scattering piece $\phi_{s}$ in a similar form:

$$
\phi_{s}(z, x)=\int \mathrm{d}^{3} y D(z, x, y) \phi_{b}(y)
$$


where $D(z, x, y)$ is the boundary-to-bulk propagator in the shockwave metric (2.3) and is inhomogeneous in the spatial coordinates. Inserting this into Eq. (3.10) we obtain the scattering piece of the classical action as

$$
S_{c l}-S_{0}=\left.\frac{N_{c}^{2}}{16 \pi^{2}} \int \mathrm{d}^{3} x \int \mathrm{d}^{3} y \phi_{b}(x) \phi_{b}(y) \frac{1}{z^{3}} \partial_{z} D(x, y, z)\right|_{z=0} .
$$

When acting on (3.19) with the functional derivatives ${ }^{10}$ in (3.5), we obtain two terms:

$$
\begin{array}{r}
\left.\frac{\delta}{\delta \phi_{b}(x) \delta \phi_{b}(y)} \int \mathrm{d}^{3} \dot{x} \int \mathrm{d}^{3} \dot{y} \phi_{b}(\dot{x}) \phi_{b}(\dot{y}) \frac{1}{z^{3}} \partial_{z} D(\dot{x}, \dot{y}, z)\right|_{z=0} \\
=\left.\frac{1}{z^{3}} \partial_{z}(D(x, y, z)+D(y, x, z))\right|_{z=0},
\end{array}
$$

which together would provide a symmetric and real expression for the current-current correlator $\Pi(x, y)$. Clearly, this is not the physical result that we are interested in. To recover the imaginary part of the retarded 2-point function, and hence the structure function $F\left(x, Q^{2}\right)$, we follow the prescription in Refs. [51,52] and drop the second term in Eq. (3.20), while multiplying the contribution of the first term by two:

$$
\Pi_{R}(x, y)=\left.\frac{N_{c}^{2}}{16 \pi^{2}} \frac{2}{z^{3}} \partial_{z} D(x, y, z)\right|_{z=0} .
$$

Note that, as it will become explicit later on, the propagator $D(x, y, z)$ is retarded with respect to the variable $x^{-}$, which plays the role of the light-cone time for the dilaton field (a left-mover). Moreover, the solution built with this propagator, cf. Eq. (3.18), is such that for large times $x^{-}$ it represents a purely 'infalling' wave, i.e. a wave which with increasing $x^{-}$propagates towards larger values of the radial dimension $z$. Thus our above prescription for keeping $D(x, y, z)$ while discarding $D(y, x, z)$ is indeed the same as the prescription for keeping the infalling solution alone, as originally formulated in Refs. [51,52].

Given $\Pi_{R}(x, y)$, the polarization function (3.4) is finally computed as

$$
\Pi\left(x, Q^{2}\right)=(\gamma / L) \Lambda^{2} \int \mathrm{d} x^{-} \mathrm{d} y^{-} \mathrm{d}^{2} x_{\perp} \mathrm{d}^{2} y_{\perp} \mathrm{e}^{i q^{+}\left(x^{-}-y^{-}\right)} \Pi_{R}(x, y),
$$

where as compared to Eq. (3.4) we have removed the Fourier transform over $x^{+}$(since this is automatically performed by working in the $q^{-}$-representation) and rewritten the measure as, e.g., $\mathrm{d} r^{-} \mathrm{d} b^{-}=\mathrm{d} x^{-} \mathrm{d} y^{-}$. One can check on the previous equations that $\Pi_{R}(x, y)$ has mass dimension 7 , hence $\Pi\left(x, Q^{2}\right)$ has dimension 4 , as it should.

In Sect. 3.3, we shall find that the structure function $F\left(x, Q^{2}\right)=\operatorname{Im} \Pi\left(x, Q^{2}\right)$ obeys the following sum-rule

$$
\lim _{Q^{2} \rightarrow \infty} \frac{\Lambda^{2}}{Q^{2}} \int_{0}^{1} \mathrm{~d} x x F\left(x, Q^{2}\right)=\frac{\pi}{20} N_{c}^{2} \Lambda^{4},
$$

which is recognized as the statement of energy-momentum conservation: this particular integral of $F\left(x, Q^{2}\right)$ singles out the coefficient in front of $T_{\mu \nu}$ (the energy-momentum tensor operator) in the operator product expansion (OPE) of the current-current correlator. The quantity in the r.h.s. of Eq. 3.23) can be recognized as the nuclear energy density in its rest frame (up to a normalization factor).

\footnotetext{
${ }^{10}$ It is now understood that the functional derivatives are defined with respect to the three-dimensional field $\phi_{b}\left(x^{-}, x_{\perp}\right)$ and for a fixed value of $q^{-}$.
} 


\subsection{Eikonal scattering}

In what follows we shall construct the scattering field $\phi_{s}$ by iterating the interaction piece in the r.h.s. of Eq. (3.12). This amounts to resumming graviton exchanges between the target and the projectile to all orders. To that aim, we need the integral version of Eq. (3.12), that is,

$$
\begin{aligned}
\phi\left(x^{-}, x_{\perp}, z\right)=\phi_{0}\left(x^{-}, x_{\perp}, z\right)+\int \frac{\mathrm{d} \dot{z}}{z^{3}} \mathrm{~d} y^{-} \mathrm{d}^{2} y_{\perp} G\left(z, \dot{z} ; x^{-}-y^{-}, x_{\perp}-y_{\perp}\right) \\
\times\left[-\left(q^{-}\right)^{2}\right] h\left(\dot{z}, y^{-}, y_{\perp}\right) \phi\left(\dot{z}, y^{-}, y_{\perp}\right),
\end{aligned}
$$

where $G\left(z, z ; ; x^{-}-y^{-}, x_{\perp}-y_{\perp}\right)$ is the bulk-to-bulk propagator obeying

$$
\left(\partial_{z}^{2}-\frac{3}{z} \partial_{z}+2 i q^{-} \partial_{-}+\nabla_{\perp}^{2}\right) G\left(z, \dot{z} ; x^{-}-y^{-}, x_{\perp}-y_{\perp}\right)=z^{3} \delta(z-\dot{z}) \delta\left(x^{-}-y^{-}\right) \delta^{(2)}\left(x_{\perp}-y_{\perp}\right),
$$

to be explicitly constructed in Appendix B. (The euclidean version of this propagator is wellknown in the literature, and will be recovered in Appendix B, but here we rather need its realtime version in light-cone coordinates and mixed Fourier representation.) Given the boundary conditions Eq. (3.14), it is clear that $G$ must vanish at the boundary. Several expressions for this propagator will be useful in what follows. For instance, the following one

$$
\begin{aligned}
G\left(z, \dot{z} ; x^{-}-y^{-}, x_{\perp}-y_{\perp}\right)=-\frac{i \Theta\left(x^{-}-y^{-}\right)}{2 q^{-}} & \int \frac{\mathrm{d}^{2} k_{\perp}}{(2 \pi)^{2}} \mathrm{e}^{i k_{\perp} \cdot\left(x_{\perp}-y_{\perp}\right)} \int_{0}^{\infty} \mathrm{d} \omega \omega \\
& \times z^{2} \mathrm{~J}_{2}(\omega z) \dot{z}^{2} \mathrm{~J}_{2}(\omega \dot{z}) \mathrm{e}^{-\frac{i\left(x^{-}-y^{-}\right)\left(\omega^{2}+k_{\perp}^{2}\right)}{2 q^{-}}}
\end{aligned}
$$

makes it clear that the propagator vanishes as $z \rightarrow 0$ and/or $\dot{z} \rightarrow 0$, and also that it is retarded with respect to the projectile time variable $x^{-}-y^{-}$. Furthermore, by using the completeness relation in Eq. (B.3) for the Bessel functions, one can show that

$$
G\left(z, \ddot{z} ; x^{-}-y^{-} \rightarrow+0, x_{\perp}-y_{\perp}\right)=-\frac{i}{2 q^{-}} z^{3} \delta(z-\dot{z}) \delta^{(2)}\left(x_{\perp}-y_{\perp}\right) .
$$

We now proceed to formally solve Eq. (3.24) via iterations. The first iteration gives

$$
\begin{array}{r}
\phi_{1}\left(z, x^{-}, x_{\perp}\right)=-\left(q^{-}\right)^{2} \int \frac{\mathrm{d} \dot{z}}{z^{3}} \mathrm{~d} \dot{x}^{-} \mathrm{d}^{2} \dot{x}_{\perp} G\left(z, \dot{z} ; x^{-}-\dot{x}^{-}, x_{\perp}-\dot{x}_{\perp}\right) \\
\times h\left(\dot{z}, \dot{x}^{-}, \dot{x}_{\perp}\right) \phi_{0}\left(\dot{z}, \dot{x}^{-}, \dot{x}_{\perp}\right),
\end{array}
$$

which we shall compactly rewrite as

$$
\phi_{1}(X)=-\left(q^{-}\right)^{2} \int \mathrm{d} \dot{X} G(X, \dot{X}) h(\dot{X}) \phi_{0}(\dot{X}) .
$$

The second iteration gives

$$
\phi_{2}(X)=\left(q^{-}\right)^{4} \int \mathrm{d} \dot{X} \ddot{X} G(X, \dot{X}) h(\dot{X}) G(\dot{X}, \ddot{X}) h(\ddot{X}) \phi_{0}(\ddot{X}),
$$

and so on. An important simplification occurs in the limit where the shockwave $h$ is treated as a $\delta$-function in $x^{-}$; then, the perturbative series produced by iterations exponentiates and yields 
an eikonal phase - a natural result at high energy. Specifically, let us write $h\left(z, x^{-}, x_{\perp}\right)=$ $\delta\left(x^{-}\right) \tilde{h}\left(z, x_{\perp}\right)$. Then from the factor $h(\dot{X}) G(\dot{X}, \ddot{X}) h\left(\ddot{X}^{\prime \prime}\right)$ in Eq. (3.30) we obtain

$$
\delta\left(\dot{x}^{-}\right) \Theta\left(\dot{x}^{-}-x^{-}\right) \delta\left(\ddot{x}^{-}\right)=\frac{1}{2} \delta\left(\dot{x}^{-}-\ddot{x}^{-}\right) \delta\left(\dot{x}^{-}\right)
$$

which after also using Eq. (3.27) gives a factor

$$
-\frac{i}{2 q^{-}} \frac{1}{2} \delta(\dot{X}-\ddot{X}) \delta\left(\dot{x}^{-}\right) .
$$

Thus Eq. (3.30) reduces to

$$
\phi_{2}(X)=-\left(q^{-}\right)^{4} \frac{i}{2 q^{-}} \int \mathrm{d} \dot{X}^{\frac{1}{2}} G(X, \dot{X}) \delta\left(\dot{x}^{-}\right) \tilde{h}^{2}(\dot{X}) \phi_{0}(\ddot{X}) .
$$

This procedure is easily generalized to higher orders: for the $k$-th iteration we deduce

$$
\frac{1}{k !}\left[-\left(q^{-}\right)^{2}\right]^{k} \tilde{h}^{k}(\dot{X})\left(-\frac{i}{2 q^{-}}\right)^{k-1},
$$

where the factorial is again generated via the product of theta functions in the propagators and delta functions in the metric field $h$. Then the sum exponentiates, as anticipated, with the following final result:

$$
\phi\left(z, x^{-}, x_{\perp}\right)=\phi_{0}\left(z, x^{-}, x_{\perp}\right)-2 q^{-} \int \frac{\mathrm{d} \dot{z}}{\dot{z}^{3}} \mathrm{~d}^{2} \dot{x}_{\perp} G\left(z, \dot{z} ; x^{-}, x_{\perp}-\dot{x}_{\perp}\right) \mathcal{T}\left(\dot{z}, \dot{x}_{\perp}\right) \phi_{0}\left(\dot{z}, 0, \dot{x}_{\perp}\right)
$$

where we have defined the scattering amplitude corresponding to a radial penetration $z$ for the dilaton and in the eikonal approximation as

$$
-i \mathcal{T}\left(z, b_{\perp}\right) \equiv 1-\exp \left[\frac{i q^{-}}{2} \tilde{h}\left(z, b_{\perp}\right)\right] .
$$

An interpretation of this quantity in the boundary gauge theory will be shortly given, following Eq. (3.41).

Using the expression of $\phi_{s}$ in Eq. (3.35) it is now straightforward to compute the DIS structure function according to Eqs. (3.2), (3.21) and (3.22). First we see that the bulk-toboundary propagator defined in equation (3.18) is given by (cf. Eq. (3.17))

$$
D(x, y, z)=-2 q^{-} \int \mathrm{d}^{2} b_{\perp} \int \frac{\mathrm{d} \dot{z}}{z^{3}} G\left(z, \dot{z} ; x^{-}, x_{\perp}-b_{\perp}\right) \mathcal{T}\left(\dot{z}, b_{\perp}\right) D_{0}\left(\dot{z},-y^{-}, b_{\perp}-y_{\perp}\right) .
$$

Clearly the integrals over $x^{-}$and $y^{-}$in Eq. (3.22) select the Fourier components with $k^{+}=q^{+}$ in both $G$ and $D_{0}$. So we are left with

$$
\begin{aligned}
\Pi\left(x, Q^{2}\right)=-\left.\frac{\gamma \Lambda^{2} N_{c}^{2}}{16 \pi^{2} L} 2 q^{-} \int \mathrm{d}^{2} x_{\perp} \mathrm{d}^{2} y_{\perp} \int \mathrm{d}^{2} b_{\perp} \int \frac{\mathrm{d} \dot{z}}{z^{3}} \frac{2}{z^{3}} \partial_{z} G\left(z, \dot{z} ; q^{+}, x_{\perp}-b_{\perp}\right)\right|_{z=0} \\
\times \mathcal{T}\left(\dot{z}, b_{\perp}\right) D_{0}\left(\dot{z}, q^{+}, b_{\perp}-y_{\perp}\right) .
\end{aligned}
$$


Using the transverse momentum space form (B.5) for the Green's function, we see that the $z$ piece in the above reads

$$
\left.\frac{2}{z^{3}} \partial_{z} G\left(z, \dot{z} ; q^{+}, k_{\perp}\right)\right|_{z=0}=-(K \dot{z})^{2} \mathrm{~K}_{2}(K \dot{z})
$$

where $K^{2}=k_{\perp}^{2}-2 q^{+} q^{-}=k_{\perp}^{2}+Q^{2}$. By also using Eq. (3.17) for $D_{0}$, we deduce

$$
\begin{array}{r}
\Pi\left(x, Q^{2}\right)=\frac{\gamma \Lambda^{2} N_{c}^{2}}{32 \pi^{2} L} 2 q^{-} \int \mathrm{d}^{2} x_{\perp} \mathrm{d}^{2} y_{\perp} \int \mathrm{d}^{2} b_{\perp} \int \frac{\mathrm{d} z}{z^{3}} \int \frac{\mathrm{d}^{2} k_{\perp}}{(2 \pi)^{2}} \mathrm{e}^{i k_{\perp} \cdot\left(x_{\perp}-b_{\perp}\right)} \\
(K z)^{2} \mathrm{~K}_{2}(K z) \mathcal{T}\left(z, b_{\perp}\right) \int \frac{\mathrm{d}^{2} q_{\perp}}{(2 \pi)^{2}} \mathrm{e}^{i q_{\perp} \cdot\left(b_{\perp}-y_{\perp}\right)}(\tilde{Q} z)^{2} \mathrm{~K}_{2}(\tilde{Q} z),
\end{array}
$$

with $\tilde{Q}^{2}=Q^{2}+q_{\perp}^{2}$. This expression simplifies drastically after performing the transverse coordinate integrals, which set $k_{\perp}=q_{\perp}=0$. Recalling the definition Eq. (3.1) we finally arrive at

$$
\Pi\left(x, Q^{2}\right)=\frac{Q^{6} \Lambda N_{c}^{2}}{32 \pi^{2} x L} \int \mathrm{d} z z \mathrm{~K}_{2}^{2}(Q z) \int \mathrm{d}^{2} b_{\perp} \mathcal{T}\left(z, b_{\perp}\right),
$$

from which the structure function $F\left(x, Q^{2}\right)$ is finally obtained by taking the imaginary part.

This result has a natural interpretation: $\mathrm{K}_{2}^{2}(Q z)$ plays the role of the wavefunction squared for a partonic fluctuation of the 'current' $J=(1 / 4) F^{2}$ which according to the UV/IR correspondence has a transverse size $r_{\perp} \sim z$. The modified Bessel function effectively restricts $r_{\perp}$ to values $r_{\perp} \sim z \lesssim 1 / Q$, as expected from the uncertainty principle. Furthermore,

$$
\sigma(z, x)=2 \int \mathrm{d}^{2} b_{\perp} \operatorname{Im} \mathcal{T}\left(z, b_{\perp}\right)
$$

is the total cross-section for the scattering between this partonic fluctuation and the 'nucleus'.

Let us finally verify that the use of the $\delta$-function approximation for the metric perturbation, and hence the eikonal approximation, are indeed justified for the problem at hand. By inspection of the previous manipulations, it is clear that the only place where the assumption that $h \propto \delta\left(x^{-}\right)$ has played a role was in using

$$
\frac{x^{-}}{2 q^{-}}\left(\omega^{2}+k_{\perp}^{2}\right) \ll 1
$$

in order to simplify the last exponential in Eq. (3.26) and thus replace the intermediate propagators in the iterative series by $\delta$-functions, cf. Eq. (3.27). In reality $x^{-}$is, of course, not strictly zero, but rather of order $L / \gamma$, which is the longitudinal width of the target in the infinite momentum frame. Since $k_{\perp}$ can never be too large (this is set by the transverse inhomogeneity in the target, so $k_{\perp} \sim \Lambda$ ), while $\omega$ is typically of order $1 / z$ (as fixed by the oscillatory behavior of the Bessel functions in Eq. (3.26)), the above inequality amounts to

$$
z^{2} \gg \frac{L}{2 q^{-} \gamma}=\frac{x \Lambda L}{Q^{2}} .
$$

Note that, in this argument, $z$ is the radial distance at some generic scattering point, e.g. $\dot{z}$ or $z$ in Eq. (3.30), and not the argument $z$ of $\phi$ in Eq. (3.35), which approaches zero when computing the structure function, cf. Eq. (3.39). As we shall shortly check, the integral over $z$ in Eq. (3.41) is dominated by $z^{2} \sim 1 / Q^{2}$; hence, the condition in Eq. (3.44) is well satisfied whenever $x \Lambda L \ll 1$, which is indeed the interesting situation (since, typically, $L \sim 1 / \Lambda$ ). 


\subsection{From single scattering to the saturation momentum}

To further compute the DIS cross-section according to Eq. (3.41) we need to specify the metric perturbation $\tilde{h}\left(z, b_{\perp}\right)$ which enters the scattering amplitude 3.36$)$. The crucial point for what follows is that, as manifest on Eq. (3.41), the DIS process is only sensitive to relatively small values of $z$, such that $z \lesssim 1 / Q \ll 1 / \Lambda$. In view of this and of the discussion in Sect. 2, it is clear that, for this purpose, it is enough to retain the dominant behavior of $\tilde{h}\left(z, b_{\perp}\right)$ near the boundary $(z \rightarrow 0)$, which for any acceptable shockwave metric scales like $z^{4} T_{--}$, cf. Eq. (2.5). Thus our formalism makes it clear that the DIS process at strong coupling involves the scattering off the nuclear energy density $T_{--}$. On the supergravity side, this scattering involves multiple graviton exchanges (here resummed in the eikonal approximation), with each factor of $z^{4}$ corresponding to one exchanged graviton. Hence, from now on we shall simply take

$$
h\left(x^{-}, b_{\perp}, z\right)=\tilde{h}\left(b_{\perp}, z\right) \delta\left(x^{-}\right)=2 \pi^{2} \frac{T_{--}}{N_{c}^{2}} z^{4},
$$

which is the approximate solution to the Einstein equation for the metric component $h$ so long as $z \Lambda \ll 1$. One can cover both type of shockwaves described in Sect. 2 by taking

$$
T_{--}\left(x^{-}, b_{\perp}\right)=\Lambda^{2} E f\left(b_{\perp} \Lambda\right) \delta\left(x^{-}\right) \equiv \tilde{T}_{--}\left(b_{\perp}\right) \delta\left(x^{-}\right),
$$

where $E$ is the total energy of the nucleus: for the type-I shockwave in Eqs. (2.15) $-(2.16)$, this reads $E=\gamma L \Lambda^{2} N_{c}^{2}$, whereas for the type-II one in Eq. 2.24) one has $E=p^{+} N_{c}^{2}$ and we identify $z_{*}=1 / \Lambda$.

Let us now consider the single scattering approximation which amounts to expanding $\mathcal{T}\left(z, b_{\perp}\right)$ in Eq. (3.36) to lowest order. By also making use of Eq. (3.45) the amplitude reads

$$
\mathcal{T}^{(1)}\left(z, b_{\perp}\right)=q^{-} \pi^{2} \frac{\tilde{T}_{--}\left(b_{\perp}\right)}{N_{c}^{2}} z^{4}
$$

which is purely real, reflecting the fact that the single graviton exchange generates no imaginary part (one cannot cut through a single graviton propagator). Note that the single graviton exchange is proportional to the energy density in the projectile and the target, and is of order zero in $N_{c}^{2}$, because the target contains $N_{c}^{2}$ degrees of freedom per unit volume (otherwise it would be suppressed like $\left.1 / N_{c}^{2}\right)$. Via the UV/IR correspondence $z \Leftrightarrow r_{\perp}$, Eq. (3.47) implies that a small dipole fluctuation of the projectile, so like a gluon-gluon pair, with transverse size $r_{\perp}$ interacts with the target with an amplitude $\mathcal{T}\left(r_{\perp}\right) \sim r_{\perp}^{4}$, which vanishes much faster at small $r_{\perp}$ than at weak coupling ${ }^{11}$ (where one rather has $\mathcal{T} \sim r_{\perp}^{2}[6]$ ). As we shall later argue, this difference is due to the fact that, at strong coupling, there are no high $-Q^{2}$ partons in the target wavefunction, that the dipole could scatter off.

In this single-scattering approximation the polarization function simplifies to

$$
\Pi^{(1)}\left(x, Q^{2}\right)=\frac{Q^{6} \Lambda N_{c}^{2}}{32 x L} \int \mathrm{d} z \mathrm{~d}^{2} b_{\perp} z \mathrm{~K}_{2}^{2}(Q z) \frac{q^{-} \tilde{T}_{--}\left(b_{\perp}\right)}{N_{c}^{2}} z^{4},
$$

\footnotetext{
${ }^{11}$ This would not be true for the shockwave metric selected in Ref. [30], and which is obtained by taking $c_{2}=0$ and $c_{1} \neq 0$ in Eq. (2.8). In that case, the DIS cross-section would involve only the first, constant, term in the small-z expansion (2.9), which via the UV/IR correspondence would imply that the scattering amplitude remains constant as $r_{\perp} \rightarrow 0$.
} 
which is purely real and hence describes only elastic scattering. The integrals in Eq. (3.48) can be exactly perform and they yield the same final result for both types of metric (for the type-I metric, the integral over $b_{\perp}$ is performed according to Eq. (2.16)), which reads

$$
\Pi^{(1)}\left(x, Q^{2}\right)=\frac{N_{c}^{2} \Lambda^{2} Q^{2}}{10 x^{2}},
$$

This is suppressed by a power of $\Lambda^{2} / Q^{2}$ with respect to the corresponding vacuum result in Eq. (3.16) and thus it is recognized as a leading-twist effect. The fact that this is purely real, i.e. the structure function $F\left(x, Q^{2}\right)$ vanishes in the leading-twist approximation, means that there are no point-like constituents in the nuclear wavefunction at strong coupling, in agreement with previous results in $[33,34,36]$. The $1 / x^{2}$ rise in Eq. (3.49), which on the supergravity side is clearly associated with the graviton exchange, can be also understood in the original gauge theory, as we explain now: in the OPE of the current-current correlator, valid at high $Q^{2}$, the leading-twist operators with $\operatorname{spin} n$ should produce contributions which behave like $1 / x^{n}$. At strong coupling, one expects all such operators to acquire large and negative anomalous dimensions [53-55], of $\mathcal{O}\left(\lambda^{1 / 4}\right)$, with the exception of the spin 2 energy-momentum tensor operator, which is protected by symmetry. Hence, the only leading-twist contribution which is expected to survive at strong coupling is the one which behaves like $1 / x^{2}$, in agreement ${ }^{12}$ with Eq. (3.49).

Although it does not contribute directly to the structure function, the single scattering contribution in Eq. (3.49) allows us to derive the sum-rule (3.23). Specifically, let us introduce the variable $\nu \equiv 1 / x$ and extend the polarization function $\Pi\left(\nu, Q^{2}\right)$ to complex values for the variable $\nu$. Then, this function is expected to be analytic everywhere in the complex $\nu$ plane except for two branch cuts along the real axis, at $\nu>1$ and $\nu<-1$ (the physical region for DIS and, respectively, the process related to DIS by crossing symmetry; see, e.g., [47]). Then, via contour integration in the complex $\nu$ plane, one can relate the behavior of this function near $\nu=0$, where the OPE applies, to integrals ('moments') of the structure function $F=\operatorname{Im} \Pi$ along the branch cuts. In the present context at strong-coupling, there is only one leading-twist contribution to $\Pi\left(\nu, Q^{2}\right)$ - the single-scattering piece $\Pi^{(1)}\left(\nu, Q^{2}\right) \propto \nu^{2} Q^{2}$, cf. Eq. (3.49) meaning that there is only one moment of the structure function which survives in the high $Q^{2}$ limit $^{13}$. Clearly, this moment is proportional to the contribution of the energy-momentum tensor (the only protected leading-twist operator) to $\Pi$. This contribution is isolated as follows:

$$
\frac{N_{c}^{2} \Lambda^{2} Q^{2}}{10}=\oint \frac{\mathrm{d} \nu}{2 \pi i} \frac{\Pi^{(1)}}{\nu^{3}} \simeq 2 \int_{1}^{\infty} \frac{\mathrm{d} \nu}{2 \pi i} \frac{2 i \operatorname{Im} \Pi}{\nu^{3}}=\frac{2}{\pi} \int_{0}^{1} \mathrm{~d} x x F\left(x, Q^{2}\right),
$$

where the contour in the first integral is a small circle surrounding the origin which is then distorted in the complex plane in such a way to wrap around the two branch cuts which give equal contributions. The approximate sign in the second equality refers to the leading-twist approximation. As anticipated, this procedure reproduces Eq. (3.23).

\footnotetext{
${ }^{12}$ Via simple dimensional arguments, one can convince oneself that the contribution of $T_{\mu \nu} \approx T_{--} \delta_{\mu-} \delta_{\nu-}$ to the OPE of $\Pi\left(x, Q^{2}\right)$ can be written as the product of $Q^{2} / x^{2}$ times a quantity which represents the average energy density per unit transverse area in the nuclear target. This quantity is of order $N_{c}^{2} \Lambda^{2}$, so the overall contribution has indeed the parametric form shown in Eq. (3.49).

${ }^{13}$ It is understood that one has to multiply the moment by the ratio $\Lambda^{2} / Q^{2}$ before taking the limit $Q^{2} \rightarrow \infty$, so like in Eq. (3.23).
} 
The dominant contribution to the DIS structure function at high $Q^{2}$ is of higher-twist order and comes from the two graviton exchange. This is easily estimated as

$$
F^{(2)}\left(x, Q^{2}\right)=\frac{\pi^{2} Q^{6} \Lambda N_{c}^{2}}{32 x L} \int \mathrm{d} z \mathrm{~d}^{2} b_{\perp} z \mathrm{~K}_{2}^{2}(Q z)\left[\frac{q^{-} \tilde{T}_{--}\left(b_{\perp}\right)}{N_{c}^{2}} z^{4}\right]^{2}=\kappa \frac{16 \pi}{7} \frac{N_{c}^{2} \Lambda^{5} L}{x^{3}}
$$

with $\kappa=\pi \Lambda^{2} \int d^{2} b_{\perp} f^{2}$; in particular, $\kappa=1 / 8$ for a type-I shockwave with the exponential profile $f=(1 / 2 \pi) \exp \left(-b_{\perp} \Lambda\right)$ and $\kappa=4 / 5$ for the type-II shockwave. Since generated by cutting in between two graviton exchanges, this contribution to the structure function can be characterized as being diffractive. Similarly, by expanding the eikonal phase in Eq. (3.36) to higher orders, one generates diffractive contributions to $F\left(x, Q^{2}\right)$ of successively higher twist order and which increase faster and faster with decreasing $x$ (as a power $1 / x^{n+1}$ for the term corresponding to $n$ graviton exchange). If one tries to use any single such a diffractive contribution within the sum-rule (3.23), the ensuing integral is ill-defined at its lower limit $x \rightarrow 0$. This reflects the fact that the expansion of the eikonal amplitude makes sense only so long as the exponent is much smaller than one. Thus, clearly, one expects a change of regime when this exponent, which is the same as the single scattering amplitude, becomes of $\mathcal{O}(1)$.

To study this change of regime, let us use Eq. (3.46) to rewrite

$$
\mathcal{T}^{(1)}=q^{-} \pi^{2} \frac{E \Lambda^{2} f\left(b_{\perp} \Lambda\right)}{N_{c}^{2}} z^{4} \sim \frac{\Lambda^{3} L f\left(b_{\perp} \Lambda\right)}{Q^{2} x} .
$$

where we have also used the fact that $z \sim 1 / Q$ together with Eq. (3.1). (This estimate applies to both types of shockwaves with the understanding that $L \sim 1 / \Lambda \sim z_{*}$ for the type-II metric.) The condition that $\mathcal{T}^{(1)}$ be of $\mathcal{O}(1)$ can be solved for $Q^{2}$ at fixed $x$ and $b_{\perp}$, thus defining the saturation momentum:

$$
Q_{s}^{2}\left(x, b_{\perp}\right)=\frac{\pi^{2} \Lambda^{3} L}{2 x} f\left(b_{\perp} \Lambda\right)
$$

where the factor $\pi^{2} / 2$ has been introduced for later convenience. Note that the transverse profile $f\left(b_{\perp} \Lambda\right)$ of the nuclear energy density directly transmits to the saturation momentum. In particular, for a shockwave which is homogeneous in the transverse impact parameter space one has $Q_{s}^{2}=\pi L \Lambda^{3} / 2 x$, in agreement with Refs. [36, 40], but in disagreement with Ref. [39], where a rather surprising result for $Q_{s}$ was reported, which becomes independent of $x$ at high energy. As it should be clear from the above analysis, and also from the corresponding ones in Refs. [36,40], the $1 / x$ rise of $Q_{s}^{2}$ at small $x$ reflects the respective behavior of the one-gravitonexchange scattering amplitude, and thus it seems unavoidable within this gauge/gravity duality context, where the high energy scattering always amounts to graviton exchanges.

Note finally that $\mathcal{T}^{(1)}\left(z \sim 1 / Q, b_{\perp}\right) \sim Q_{s}^{2}\left(x, b_{\perp}\right) / Q^{2}$, which reaches its maximal value at the center of the 'nucleus' $\left(b_{\perp}=0\right)$. This makes it clear that the 'twist' expansion of the eikonal phase is an expansion in powers of $Q_{s}^{2}(x, 0) / Q^{2}$ and is appropriate in the high- $Q^{2}$ regime at $Q^{2} \gg Q_{s}^{2}(x, 0)$. The opposite regime, at $Q^{2} \lesssim Q_{s}^{2}(x, 0)$, or equivalently $x \leq x_{s}(Q)$ where,

$$
x_{s}(Q)=\frac{\pi^{2} \Lambda^{3} L}{2 Q^{2}} f(0),
$$

will be studied in the next subsection. 


\subsection{Structure function at saturation}

Having identified the saturation momentum which marks the borderline between weak and strong scattering, we shall now compute the structure function in the 'saturation region' at $Q^{2} \lesssim Q_{s}^{2}(x, 0)$. This will allow us to understand how the energy-momentum sum rule (3.23) is satisfied and speculate about a possible partonic interpretation.

Let us start by rewriting the scattering amplitude defined in Eq. (3.36) in terms of the saturation momentum introduced in the previous subsection. It reads

$$
\mathcal{T}\left(z, x, b_{\perp}\right)=i\left\{1-\exp \left[i Q^{2} Q_{s}^{2}\left(x, b_{\perp}\right) z^{4}\right]\right\}
$$

In this high energy, or relatively low $Q^{2}$ regime, that we are interested in, there is a central region of the nucleus which looks 'black' to the projectile. That is, for any given $z$ there is a 'black disk' radius $R_{\mathrm{b}}(z, Q, x)$ such that, for all $b_{\perp} \lesssim R_{\mathrm{b}}$ the eikonal phase in Eq. 3.55) can be neglected since rapidly oscillating and the scattering amplitude becomes purely imaginary with a magnitude equal to one, meaning that the dilaton component at radial distance $z$ is completely absorbed in the shockwave. Recalling also Eq. (3.53), we see that this black disk radius is determined by

$$
\frac{f\left(R_{\mathrm{b}} \Lambda\right)}{f(0)}=\frac{1}{Q^{2} Q_{s}^{2}(x, 0) z^{4}}
$$

For given $x$ and $Q^{2}$, a black disk exists at the center of the 'nucleus' $\left(b_{\perp}=0\right)$ only for those components of the dilaton which have penetrated far enough in the radial direction, namely up to $z \geq z_{0}$ with $z_{0}=1 / \sqrt{Q Q_{s}(x, 0)}$. Physically, this means that the transverse size $r_{\perp} \sim z$ of the partonic fluctuations of the projectile should be large enough for their color dipolar interactions in the target to be strong. Since on the other hand $z$ is restricted to $z \lesssim 1 / Q$ by the dilaton wavefunction, it is clear that a black disk can form only when the energy is high enough for the condition $Q^{2} \lesssim Q_{s}^{2}(x, 0)$ to be satisfied. Once that the black disk appears at the center of the 'nucleus' (for a given $z \geq z_{0}$ ) it rapidly occupies the whole central region of the shockwave, which is essentially flat in $b_{\perp}$. When further increasing the energy, the black disk extends al larger values of $b_{\perp}$, at a rate which depends upon the transverse profile of the shockwave.

For example, for a type-II shockwave the black disk radius will be given by

$$
\left(R_{\mathrm{b}} \Lambda\right)^{2}=\left[Q^{2} Q_{s}^{2}(x, 0) z^{4}\right]^{1 / 3}-1 .
$$

This exhibits a rapid increase with the energy, proportional to $(1 / x)^{1 / 3}$, which reflects the power law tail in $b_{\perp}$ of the respective energy density profile. On the contrary, a type-I shockwave with an exponential tail at large $b_{\perp}$ will have a black disk radius which at high energy increases with $1 / x$ only logarithmically, that is

$$
\left(R_{\mathrm{b}} \Lambda\right)^{2}=\ln ^{2}\left[Q^{2} Q_{s}^{2}(x, 0) z^{4}\right] .
$$

For high enough energy, the total cross section (3.42) for a given value of $z$ will be proportional to the area of the respective black disk. (Indeed, outside the black disk, at $b_{\perp} \gg R_{\mathrm{b}}(z, Q, x)$, the amplitude is very small and rapidly decreasing with $b_{\perp}$.) So, clearly, the cross-section associated to the type-II shockwave will rise like a power of the energy, in violation of the 
Froissart bound, whereas that corresponding to a type-I shockwave with exponential tail will saturate the Froissart bound at high enough energy. In that sense, the type-II shockwave is a more realistic model for a nucleus in QCD. In fact, the above mechanism for the emergence of the Froissart bound is exactly the same as expected in QCD: the competition between the power-law increase of the scattering amplitude with the energy at a given $b_{\perp}$ and the exponential decrease of the nuclear matter distribution at large $b_{\perp}$.

The previous considerations lead to the following expression for the structure function at $Q^{2}<Q_{s}^{2}(x, 0):$

$$
F\left(x, Q^{2}\right)=\frac{N_{c}^{2}}{16 \pi} \frac{Q^{6}}{x \Lambda L} \int_{z_{0}}^{\infty} \mathrm{d} z z \mathrm{~K}_{2}^{2}(Q z)\left[R_{\mathrm{b}}(z, Q, x) \Lambda\right]^{2} .
$$

The Bessel function will effectively cut off all contributions coming from $z \gtrsim 1 / Q$, so that the integration in Eq. (3.59) is in practice supported only in the interval

$$
\frac{1}{\sqrt{Q Q_{s}(x, 0)}} \lesssim z \lesssim \frac{1}{Q}
$$

However, the dilaton wavefunction diverges strongly at small $z$, more precisely $\mathrm{K}_{2}^{2}(Q z) \simeq$ $4 / Q^{4} z^{4}$. Thus for any profile $f\left(b_{\perp} \Lambda\right)$ which leads to a black disk area $\left(R_{\mathrm{b}} \Lambda\right)^{2}$ larger than $z^{2}$ at $z \ll 1$, therefore including the two cases in Eqs. (3.57) and (3.58), the integration is dominated by its lower limit. This comes as a surprise, since one might have expected to dominant contribution to come from $z \sim 1 / Q$, as happens in the case of the $\mathcal{R}$-current (see Sect. $⿴$ below), and also in perturbative QCD (after identifying $z$ with the size of the dipole fluctuation of the virtual photon). Coming back to the case under study, we see that for $z \sim z_{0}$ the black disk area $\left(R_{\mathrm{b}} \Lambda\right)^{2}$ is a number of $\mathcal{O}(1)$ and thus the integration is simply proportional to $1 / Q^{4} z_{0}^{2}=Q_{s}(x, 0) / Q^{3}$. So, quite remarkably, the structure function for the dilaton DIS is not sensitive to the expansion of the black disk with increasing energy - rather, it is controlled by the central part of the black disk at $b_{\perp} \lesssim 1 / \Lambda-$, and hence it is not affected, e.g., by the violation of the Froissart bound in the case of the type-II shockwave. This should be contrasted with the corresponding situation for the $\mathcal{R}$-current, to be described in Sect. 6 .

As we show in Appendix [), deeply at saturation $\left(Q^{2} \ll Q_{s}^{2}(x, 0)\right)$, we can also determine the precise normalization of the structure function, and we finally have

$$
F\left(x, Q^{2}\right)=\kappa \frac{N_{c}^{2}}{16 \sqrt{2 \pi}} \frac{Q^{4}}{x \Lambda L} \frac{Q_{s}(x, 0)}{Q},
$$

with $\kappa$ a number which depends only on the profile of the shockwave under consideration.

It is straightforward to check that in the transition region at $Q \sim Q_{s}(x)$, or equivalently $x \sim x_{s}(Q)$, the above result in Eq. (3.61) is parametrically consistent with the respective extrapolation of the dominant result at large- $x$, i.e. the 2-graviton exchange contribution in Eq. (3.51):

$$
x_{s} F_{s}\left(x_{s}, Q^{2}\right) \sim \frac{Q^{4} N_{c}^{2}}{\Lambda L} \sim x_{s} F^{(2)}\left(x_{s}, Q^{2}\right) .
$$

Of course, for $x \sim x_{s}(Q)$ all the terms in the twist-expansion become parametrically of the same order and our various approximations fail to apply there, except for parametric estimates. 
By using these estimates, one can now verify that the energy-momentum sum rule (3.23) is indeed satisfied parametrically. The integral over $x$ in the l.h.s. of Eq. (3.23) is dominated by $x \sim x_{s}(Q)$, since for $x \gg x_{s}$ the 2-graviton exchange contribution in Eq. (3.51) is rapidly decreasing with $x$, whereas for $x \lesssim x_{s}$, we have $x F\left(x, Q^{2}\right) \sim 1 / \sqrt{x}$. Thus, by making use of Eqs. (3.62) and (3.54) one can write

$$
\int_{0}^{1} \mathrm{~d} x x F\left(x, Q^{2}\right) \sim x_{s}^{2} F\left(x_{s}, Q^{2}\right) \sim \frac{Q^{4} N_{c}^{2} x_{s}}{\Lambda L} \sim Q^{2} N_{c}^{2} \Lambda^{2} .
$$

Let us conclude this discussion by suggesting a possible partonic interpretation for the previous results. The standard OPE analysis for the correlator (3.3) shows that, in the weak coupling regime and for sufficiently high $Q^{2}$, the quantity $x F\left(x, Q^{2}\right) / Q^{2}$ has the meaning of the gluon distribution in the target (since gluons are the partons which directly couple to the relevant operator $\left.J=(1 / 4) F^{2}\right)$. Namely, it is proportional with the number of gluons per unit transverse area having longitudinal momentum fraction $x$ and transverse momenta $k_{\perp} \lesssim Q$. Then what about the previous results at strong coupling ? Since the energy-momentum sum-rule is controlled by $x \sim x_{s}(Q)$, it is natural to look at Eq. (3.62), which implies $x_{s} F_{s}\left(x_{s}, Q^{2}\right) / Q^{2} \sim Q^{2} N_{c}^{2}$. As we shall argue in more detail in Sect. 4.3, where a similar result occurs in the context of the $\mathcal{R}$-current, this estimate is indeed consistent with a partonic picture, in which the partons (here, gluons) are distributed along the saturation line with occupation numbers of order one

14. One the other hand, there are clearly no partons at $x \gg x_{s}(Q)$, as obvious from the lack of leading-twist contributions to $F\left(x, Q^{2}\right)$ in that region.

\section{DIS off the shockwave: the $\mathcal{R}$-current}

Let us now turn to the problem of deep inelastic scattering of an $\mathcal{R}$-current $J_{\mu}$ off the shockwave. As discussed in the beginning of Sect. 解 this is a conserved current in $\mathcal{N}=4 \mathrm{SYM}$ which is carried by the fermionic and scalar fields. It is a bilinear in both of these types of fields, whose precise form is not necessary for our discussion (it is given, for example, in [34]), and it has mass dimension 3 .

\subsection{General equations: from the 5D action to the polarization tensor}

We will be interested in calculating the polarization tensor

$$
\Pi^{\mu \nu}(q)=\frac{\gamma \Lambda^{2}}{L} \int \mathrm{d}^{4} r \mathrm{~d} b^{-} \mathrm{d}^{2} b_{\perp} \mathrm{e}^{i q \cdot r} i \Theta\left(x_{0}\right)\left\langle\left[J^{\mu}(b+r / 2), J^{\nu}(b-r / 2)\right]\right\rangle,
$$

which is the analogous to Eq. (3.4) and where the notation is the same as in the previous section. The bulk field dual to the $\mathcal{R}$-current $J_{\mu}$ is the $\mathrm{SO}(6)$ gauge field $A^{m}$, where we have suppressed the fixed color index of this vector field corresponding to the fixed index of the $\mathcal{R}$-current. To compute $\Pi^{\mu \nu}$ (see Eq. (4.3) below), we need only the quadratic part of the respective Yang-Mills action in the $\mathrm{AdS}_{5}$ background. It reads

$$
S=-\frac{N_{c}^{2}}{64 \pi^{2} R} \int \mathrm{d}^{4} x \mathrm{~d} z \sqrt{-g} g^{m p} g^{n q} F_{m n} F_{p q},
$$

\footnotetext{
${ }^{14}$ At this stage, it might be useful to remember that, in the saturation region at $x \leq x_{s}(Q)$, the partonic interpretation of the DIS structure functions does not rely on the validity of the twist expansion (and the dominance of the twist-two operators) not even at weak coupling [6].
} 
with $F_{m n}=\partial_{m} A_{n}-\partial_{n} A_{m}$. According to the AdS/CFT correspondence the 2-point function of the current appearing in the integrand Eq. (4.1) is equal to

$$
\Pi^{\mu \nu}(x, y)=\left.\frac{\delta^{2} S_{\mathrm{cl}}}{\delta \mathcal{A}_{\mu}(x) \delta \mathcal{A}_{\nu}(y)}\right|_{\mathcal{A}_{\mu}=0} .
$$

In the above, $S_{\mathrm{cl}}$ is the action evaluated on the solution to the equations of motion

$$
\partial_{m}\left(\sqrt{-g} g^{m p} g^{n q} F_{p q}\right)=0
$$

i.e. the Maxwell equations in curved space-time, with the following boundary conditions

$$
\lim _{z \rightarrow 0} A_{\mu}(z, x)=\mathcal{A}_{\mu}(x) \text { and } \lim _{z \rightarrow 0} A_{z}(z, x)=0 .
$$

It will be convenient to adopt the gauge condition $A_{z}=0$. Furthermore, as in the case of the dilaton, we shall assume that the boundary field is a plane wave of the form

$$
\mathcal{A}_{\mu}(x)=\mathrm{e}^{-i\left(q^{-} x^{+}+q^{+} x^{-}\right)} \tilde{\mathcal{A}}_{\mu},
$$

so that the components of $\tilde{\mathcal{A}}_{\mu}$ are pure numbers. Now let us integrate Eq. (4.2) by parts. Using the Maxwell equations (4.4) only the boundary term survives to give

$$
S_{\mathrm{cl}}=-\frac{N_{c}^{2}}{32 \pi^{2} R} \int \mathrm{d}^{4} x \mathrm{~d} z \partial_{m}\left(\sqrt{-g} g^{m p} g^{n q} F_{p q} A_{n}\right) .
$$

Substitution of our shockwave metric into the above leads to

$$
S_{\mathrm{cl}}=\left.\frac{N_{c}^{2}}{32 \pi^{2}} \int \mathrm{d}^{4} x \frac{1}{z}\left(-A_{+} A_{-}^{\prime}-A_{-} A_{+}^{\prime}+A_{i} A_{i}^{\prime}\right)\right|_{z=0}
$$

where a prime represents differentiation with respect to $z$, the index $i=1,2$ refers to the transverse components and we have dropped a term $-h A_{+} A_{+}^{\prime}$ inside the parenthesis which does not contribute since $h \propto z^{4}$ for small $z$.

For simplicity, from now on we will restrict to the case where the fields are independent of $x_{\perp}$, which is the situation when the shockwave is homogeneous in transverse directions: $\partial_{i} h=0$. Furthermore, since the shockwave metric in Eq. (2.3) does not depend on $x^{+}$, we can restrict ourselves to a single Fourier mode of the gauge field as follows

$$
A_{\mu}\left(z, x^{+}, x^{-}\right)=\mathrm{e}^{-i q^{-} x^{+}} A_{\mu}\left(z, x^{-}\right) .
$$

The Maxwell equations for $n=-, z, i$ become

$$
\begin{aligned}
& \left(z \partial_{z} z^{-1} \partial_{z}+i q^{-} \partial_{-}\right) A_{+}=\left(q^{-}\right)^{2} A_{-}, \\
& \left(\partial_{-}-i q^{-} h\right) A_{+}^{\prime}-=i q^{-} A_{-}^{\prime}, \\
& \left(z \partial_{z} z^{-1} \partial_{z}+2 i q^{-} \partial_{-}\right) A_{i}=-\left(q^{-}\right)^{2} h A_{i} .
\end{aligned}
$$

Notice that in the vacuum case $h=0$, the $x^{-}$dependence of the field is that of a plane wave, that is $\mathrm{e}^{-i q^{+} x^{-}}$. The first two equations are coupled. In order to solve them we differentiate 
(4.10) with respect to $z$ and we make use of (4.11) to arrive at the differential equation which determines $A_{+}^{\prime}$ :

$$
\left(\partial_{z} z \partial_{z} z^{-1}+2 i q^{-} \partial_{-}\right) A_{+}^{\prime}=-\left(q^{-}\right)^{2} h A_{+}^{\prime} .
$$

As in the dilaton case, we separate the total field into a vacuum piece and a scattering piece

$$
A_{\mu}\left(z, x^{-}\right)=A_{\mu}^{(0)}\left(z, x^{-}\right)+A_{\mu}^{(s)}\left(z, x^{-}\right)
$$

with $A_{\mu}^{(0)}\left(z, x^{-}\right)$satisfying the vacuum version of the Maxwell equations which are obtained by setting $h=0$. We shall construct $A_{\mu}\left(z, x^{-}\right)$so that its boundary condition at $z=0$ is fully encoded in the vacuum field, that is

$$
\lim _{z \rightarrow 0} A_{\mu}^{(0)}\left(z, x^{-}\right)=\mathcal{A}_{\mu}\left(x^{-}\right) \text {and } \lim _{z \rightarrow 0} A_{\mu}^{(s)}\left(z, x^{-}\right)=0 .
$$

Now we define the boundary to bulk propagator $D^{\mu \nu}$ according to

$$
A_{\mu}^{(s)}\left(z, x^{-}\right)=\int \mathrm{d} y^{-} D_{\mu}^{\nu}\left(z, x^{-}, y^{-}\right) \mathcal{A}_{\nu}\left(y^{-}\right)
$$

which must be determined by solving the equations of motion. Then the contribution of the scattering piece to the classical action Eq. (4.8) is obtained from $-A_{+}^{(0)} A_{-}^{\prime(s)}-A_{-}^{(0)} A_{+}^{(s)}+A_{i}^{(0)} A_{i}^{\prime(s)}$ and reads

$$
S_{\mathrm{cl}}-S_{0}=\left.V \frac{N_{c}^{2}}{32 \pi^{2}} \int \mathrm{d} x^{-} \mathrm{d} y^{-} \mathcal{A}_{\mu}\left(x^{-}\right) \mathcal{A}_{\nu}\left(y^{-}\right) \frac{1}{z} \partial_{z} D^{\mu \nu}\left(z, x^{-}, y^{-}\right)\right|_{z=0},
$$

with the volume factor $V$ arising from the integration over $x^{+}$and $x_{\perp}$. Functional differentiation according to Eq. (4.3) and use of the same prescription as the one used in the dilaton case (cf. the discussion just after Eq. (3.20)) leads to ${ }^{15}$

$$
\Pi_{\mathrm{R}}^{\mu \nu}=\left.\frac{1}{V} \frac{N_{c}^{2}}{16 \pi^{2}} \frac{1}{z} \partial_{z} D^{\mu \nu}\left(z, x^{-}, y^{-}\right)\right|_{z=0} .
$$

Substituting into Eq. (4.1) we finally arrive at

$$
\Pi^{\mu \nu}(q)=\left.\frac{\gamma}{L} \frac{N_{c}^{2}}{16 \pi^{2}} \int \mathrm{d} x^{-} \mathrm{d} y^{-} \mathrm{e}^{i q^{+}\left(x^{-}-y^{-}\right)} \frac{1}{z} \partial_{z} D^{\mu \nu}\left(z, x^{-}, y^{-}\right)\right|_{z=0} .
$$

Let us notice here that not all the components of the propagator $D^{\mu \nu}$, and therefore of the tensor $\Pi^{\mu \nu}$, are independent. In fact, only two components are independent since $\Pi^{\mu \nu}$ can be decomposed as

$$
\Pi^{\mu \nu}(q)=\left(\eta^{\mu \nu}-\frac{q^{\mu} q^{\nu}}{Q^{2}}\right) \Pi_{1}\left(x, Q^{2}\right)+\left(p^{\mu}-\frac{p \cdot q}{Q^{2}} q^{\mu}\right)\left(p^{\nu}-\frac{p \cdot q}{Q^{2}} q^{\nu}\right) \Pi_{2}\left(x, Q^{2}\right),
$$

which follows from general symmetries (namely the current conservation together with the timereversal symmetry $\Pi^{\mu \nu}(q)=\Pi^{\mu \nu}(-q)$ ) and can be explicitly verified by calculating one by one all the components of the propagator $D^{\mu \nu}$. Hence we will just need to determine $\Pi^{i i}$ and, for example, $\Pi^{--}$, since the remaining non-vanishing components will be then determined as

$$
\Pi^{++}=\frac{Q^{4}}{4\left(q^{-}\right)^{4}} \Pi^{--} \quad \text { and } \quad \Pi^{+-}=\Pi^{-+}=\frac{Q^{2}}{2\left(q^{-}\right)^{2}} \Pi^{--} .
$$

\footnotetext{
${ }^{15}$ Notice that there is a factor $1 / V$ for each functional differentiation since $\delta / \delta \mathcal{A}(x)=(1 / V) \delta / \delta \mathcal{A}\left(x^{-}\right)$.
} 


\subsection{The classical solution}

The solution to the equations of motion is constructed in the same way as in the dilaton case. Resumming the graviton exchanges to all orders we find for the scattering part of the transverse field

$$
A_{i}^{(s)}\left(x^{-}, z\right)=-2 q^{-} \int \frac{\mathrm{d} \dot{z}}{\dot{z}} G_{\mathrm{T}}\left(z, \dot{z} ; x^{-}\right) \mathcal{T}(\dot{z}) A_{i}^{(0)}(0, \dot{z}),
$$

where the scattering amplitude $\mathcal{T}(\dot{z})$ has already been defined in Eq. (3.36) and with the Green's function $G_{\mathrm{T}}$ satisfying in momentum space

$$
\left(z \partial_{z} z^{-1} \partial_{z}-K^{2}\right) G_{\mathrm{T}}\left(z, \dot{z} ; K^{2}\right)=z \delta(z-\dot{z})
$$

with the notation $K^{2}=-2 k^{+} q^{-}$. The precise form of the Green's function is given in Appendix $\mathrm{B}$. Now we can see that the respective boundary to bulk propagator reads

$$
D^{i i}\left(z, x^{-}, y^{-}\right)=-2 q^{-} \int \frac{\mathrm{d} \dot{z}}{\dot{z}} G_{\mathrm{T}}\left(z, \dot{z} ; x^{-}\right) \mathcal{T}(\dot{z}) D_{0}^{i i}\left(\dot{z},-y^{-}\right)
$$

where $D_{0}^{i i}$ is the corresponding boundary to bulk propagator in the vacuum and in momentum space is given by

$$
D_{0}^{i i}(z, K)=K z \mathrm{~K}_{1}(K z)
$$

Substituting the above into Eq. (4.19) we can immediately perform the integrations over the longitudinal coordinates which will select the $q^{+}$component of both the Green's function $G_{\mathrm{T}}$ and the propagator $D_{0}^{i i}$. We then have (recall that $Q^{2}=-2 q^{+} q^{-}>0$ )

$$
\Pi^{i i}=-\left.\frac{N_{c}^{2}}{16 \pi^{2}} \frac{Q^{2}}{x \Lambda L} \int \frac{\mathrm{d} \dot{z}}{\dot{z}} \frac{1}{z} \partial_{z} G_{\mathrm{T}}\left(z, \dot{z} ; Q^{2}\right)\right|_{z=0} \mathcal{T}(\dot{z}) D_{0}^{i i}(\dot{z}, Q) .
$$

Finally by making use of

$$
-\left.\frac{1}{z} \partial_{z} G_{\mathrm{T}}\left(z, \dot{z} ; Q^{2}\right)\right|_{z=0}=Q \dot{z} \mathrm{~K}_{1}(Q \dot{z})
$$

and Eq. (4.25), and changing the name of the integration variable from $z$ to $z$, we arrive at

$$
\Pi^{i i}=\frac{N_{c}^{2}}{16 \pi^{2}} \frac{Q^{4}}{x \Lambda L} \int \mathrm{d} z z \mathrm{~K}_{1}^{2}(Q z) \mathcal{T}(z) .
$$

We recognize here a structure similar to that in Eq. (3.41): once again, the Bessel function $\mathrm{K}_{1}^{2}(Q z)$ plays the role of the wavefunction squared for the partonic fluctuation of the $\mathcal{R}$-current (namely, a fluctuation with transverse size $\Delta x_{\perp} \sim z$ ). Interestingly, it is the same Bessel function which determines the wavefunction of the quark-antiquark fluctuation of a space-like photon in lowest-order perturbative QCD. The same remark applies to the structure of $\Pi^{--}$, that we now compute.

Namely, the scattering part of the $A_{+}$component of the gauge field is found as

$$
A_{+}^{\prime(s)}\left(x^{-}, z\right)=-2 q^{-} \int \frac{\mathrm{d} \dot{z}}{\dot{z}} G_{\mathrm{L}}\left(z, \dot{z} ; x^{-}\right) \mathcal{T}(\dot{z}) A_{+}^{\prime(0)}(0, \dot{z}),
$$


with the Green's function $G_{\mathrm{L}}$ satisfying in momentum space

$$
\left(\partial_{z} z \partial_{z} z^{-1}-K^{2}\right) G_{\mathrm{L}}\left(z, \dot{z} ; K^{2}\right)=z \delta(z-\dot{z})
$$

and again the precise form of the Green's function is given in Appendix B. Now we need to be careful when we express the vacuum field $A_{+}^{\prime(0)}(0, \dot{z})$ in terms of the boundary values. We have

$$
A_{+}^{\prime(0)}\left(x^{-}, z\right)=\int \mathrm{d} y^{-} \partial_{z} D_{0}^{-\mu}\left(z, x^{-}, y^{-}\right) \mathcal{A}_{\mu}\left(y^{-}\right),
$$

where the upper index $\mu$ can be either + or - . Both components of the boundary to bulk propagator $\partial_{z} D_{0}^{-\mu}\left(z, x^{-}, y^{-}\right)$satisfy the vacuum version of Eq. (4.13) and in momentum space they are given by

$$
\partial_{z} D_{0}^{-\mu}(z, K)=\frac{1}{2} K^{2} z \mathrm{~K}_{0}(K z) c^{\mu} \quad \text { with } \quad c^{+}=1 \quad \text { and } \quad c^{-}=\frac{2\left(q^{-}\right)^{2}}{Q^{2}} .
$$

The coefficients $c^{\mu}$ have been determined by plugging the above equations into the left hand side of Eq. (4.10) and then evaluating both sides of that equation on the boundary. Since we would like to calculate $\Pi^{--}$we shall need here only the $D_{0}^{--}$component, but it is already obvious from the above equation why the ratio $\Pi^{+-} / \Pi^{--}$is given by the second equation in Eq. (4.21). Now we are ready to give the boundary to bulk propagator which reads

$$
\partial_{z} D^{--}\left(z, x^{-}, y^{-}\right)=-2 q^{-} \int \frac{\mathrm{d} \dot{z}}{\dot{z}} G_{\mathrm{L}}\left(z, \dot{z} ; x^{-}\right) \mathcal{T}(\dot{z}) \partial_{\dot{z}} D_{0}^{--}\left(\dot{z},-y^{-}\right) .
$$

We proceed as before and we plug into Eq. (4.19) to obtain

$$
\Pi^{--}=-\left.\frac{N_{c}^{2}}{16 \pi^{2}} \frac{Q^{2}}{x \Lambda L} \int \frac{\mathrm{d} \dot{z}}{\dot{z}} \frac{1}{z} G_{\mathrm{L}}\left(z, \dot{z} ; Q^{2}\right)\right|_{z=0} \mathcal{T}(\dot{z}) \partial_{\dot{z}} D_{0}^{i i}(\dot{z}, Q) .
$$

Using $-\left.(1 / z) G_{\mathrm{L}}\left(z, \dot{z} ; Q^{2}\right)\right|_{z=0}=\dot{z} \mathrm{~K}_{0}(Q \dot{z})$ and then letting $\dot{z} \rightarrow z$ we finally obtain

$$
\frac{Q^{2}}{\left(q^{-}\right)^{2}} \Pi^{--}=\frac{N_{c}^{2}}{16 \pi^{2}} \frac{Q^{4}}{x \Lambda L} \int \mathrm{d} z z \mathrm{~K}_{0}^{2}(Q z) \mathcal{T}(z)
$$

\subsection{Structure functions and their partonic interpretation}

We are finally in a position to calculate the Lorentz invariant structure functions $F_{1}$ and $F_{2}$. In general they are given by

$$
F_{1}=\frac{1}{2 \pi} \operatorname{Im} \Pi_{1}, \quad F_{2}=-\frac{p \cdot q}{2 \pi} \operatorname{Im} \Pi_{2} .
$$

Focusing on the (--) and (ii) components in (4.20) we can express $\Pi_{1}$ and $\Pi_{2}$, and therefore the structure functions, in terms of the components $\Pi^{--}$and $\Pi^{i i}$ that we have already calculated. It

is also customary and convenient to introduce the transverse and longitudinal structure functions $F_{\mathrm{T}}$ and $F_{\mathrm{L}}$. Let us summarize here our results for all these various structure functions:

$$
\begin{aligned}
& F_{\mathrm{T}}=2 x F_{1}=\frac{x}{\pi} \operatorname{Im} \Pi^{i i}=\frac{N_{c}^{2}}{16 \pi^{3}} \frac{Q^{4}}{\Lambda L} \int \mathrm{d} z z \mathrm{~K}_{1}^{2}(Q z) \mathcal{T}(z), \\
& F_{\mathrm{L}}=\frac{x}{\pi} \operatorname{Im} \frac{Q^{2}}{\left(q^{-}\right)^{2}} \Pi^{--}=\frac{N_{c}^{2}}{16 \pi^{3}} \frac{Q^{4}}{\Lambda L} \int \mathrm{d} z z \mathrm{~K}_{0}^{2}(Q z) \mathcal{T}(z), \\
& F_{2}=F_{\mathrm{T}}+F_{\mathrm{L}} .
\end{aligned}
$$


These results are in agreement with those in Ref. [40]; there is in fact a mismatch by an overall factor $\Lambda L$, but this is presumably related to the use of different conventions in the normalization of the current-current correlator.

We have already seen that the scattering amplitude $\mathcal{T}$ that emerged during the calculation is the same as the one appeared in the dilaton case. Thus the saturation scale will also be the same in the case we are currently considering. This is natural since the saturation scale is a property of the nuclear target and hence it should be the same whether we probe it with the gluon current or with the $\mathcal{R}$-current. Recalling that $Q_{s}^{2}=\pi \Lambda^{3} L / 2 x$ (in a homogeneous situation) we can write the amplitude $\mathcal{T}$ as

$$
\mathcal{T}(z, Q)=i\left[1-\exp \left(i Q^{2} Q_{s}^{2} z^{4}\right)\right]
$$

Since it not possible to obtain an analytic expression for the structure functions for arbitrary value of $x$ and $Q^{2}$, we shall separately consider the two limiting cases.

(i) For $Q \gg Q_{s}(x)$ (or, equivalently, $x \gg x_{s}(Q)$, with $x_{s}(Q)$ defined in Eq. (3.54)) we need to expand the exponential in (4.40) to second order since the first order term will not contribute to the imaginary part of the tensor $\Pi^{\mu \nu}$. The upper limit in the $z$-integration is $1 / \sqrt{Q Q_{s}} \gg 1 / Q$, so it can be set equal to $\infty$. We find

$$
F_{\mathrm{T}}=\frac{16 N_{c}^{2}}{7 \pi} \frac{\Lambda^{2}}{x} \frac{Q_{s}^{2}}{Q^{2}}, \quad F_{\mathrm{L}}=\frac{64 N_{c}^{2}}{35 \pi} \frac{\Lambda^{2}}{x} \frac{Q_{s}^{2}}{Q^{2}} \quad \text { and } \quad F_{2}=\frac{144 N_{c}^{2}}{35 \pi} \frac{\Lambda^{2}}{x} \frac{Q_{s}^{2}}{Q^{2}} .
$$

As in the dilaton case, these contributions are of higher-twist order (in the context of the $\mathcal{R}$ current, a leading-twist contribution would be independent of $Q^{2}$ at large $Q^{2}$, up to logarithms), which reflects the absence of point-like constituents in the nucleus.

(ii) For $Q \ll Q_{s}(x)$ (or, equivalently, $x \ll x_{s}(Q)$ ), we set the exponential in (4.40) equal to zero and we restrict the integration to $z \gtrsim 1 / \sqrt{Q Q_{s}}$. In the longitudinal sector, and since $\mathrm{K}_{0}$ diverges only logarithmically at small $z$, the integration is dominated by the region $z \sim 1 / Q$, so that the lower limit can be safely set equal to zero. This is not the case in the transverse sector where the integration is sensitive to small values. Since $\mathrm{K}_{1}(Q z) \simeq 1 / Q z$ for small $z$, there is a logarithmically enhanced contribution when compared to $F_{\mathrm{L}}$. Therefore the transverse sector dominates in $F_{2}$, albeit this dominance is only logarithmic. We get

$$
F_{2} \simeq F_{\mathrm{T}}=\frac{N_{c}^{2}}{64 \pi^{3}} \frac{Q^{2}}{\Lambda L} \ln \frac{Q_{s}^{2}}{Q^{2}} \quad \text { and } \quad F_{\mathrm{L}}=\frac{N_{c}^{2}}{32 \pi^{3}} \frac{Q^{2}}{\Lambda L} .
$$

Remarkably, the structure functions at low $Q^{2}$ have the same parametric form as for a proton in QCD at weak coupling, except for the replacement of the baryonic color factor $N_{c}$ corresponding to a proton by a factor $N_{c}^{2}$ corresponding to the density of degrees of freedom in the shockwave.

Even though the above expressions in Eqs. (4.41) and (4.42) are strictly valid for $Q \gg Q_{s}$ and $Q \ll Q_{s}$ respectively, they become of the same order when extrapolated to $Q \sim Q_{s}$ and therefore they can be parametrically trusted even for $Q$ around $Q_{s}$. This also means that for $Q \sim Q_{s}$ the transverse and longitudinal structure functions are of the same order.

So far, our analysis in the $\mathcal{R}$-current case has been done for a homogeneous, in the transverse space, shockwave. However, given the close analogy of the structure functions in Eqs. (4.37) and (4.38) to the dilaton structure function in Eq. (3.41) and the common form of the scattering 
amplitude in the two cases, it is not unreasonable to extend the discussion of Sect. 3.4 to the problem of an inhomogeneous shockwave probed by an $\mathcal{R}$-current. In such a situation, and according to Eq. (3.59), we would write the transverse and longitudinal structure functions at saturation $\left(Q^{2} \ll Q_{s}^{2}(x, 0)\right)$ as

$$
F_{\mathrm{T}, \mathrm{L}}\left(x, Q^{2}\right)=\frac{N_{c}^{2}}{8 \pi^{2}} \frac{Q^{4}}{\Lambda L} \int_{z_{0}}^{\infty} \mathrm{d} z z \mathrm{~K}_{1,0}^{2}(Q z)\left[R_{\mathrm{b}}(z, Q, x) \Lambda\right]^{2},
$$

with $R_{\mathrm{b}}$ and $z_{0}$ as in Sect. 3.4. But, in contrast to the dilaton case, the $z$-integration is now dominated by $z \sim 1 / Q$ in the longitudinal sector, while it is only logarithmically sensitive to the lower limit in the transverse sector (like in the homogeneous case), a behavior similar to the one in QCD. For the exponential profile leading to the black disk radius in Eq. (3.58), we find

$$
F_{2} \simeq F_{\mathrm{T}}=\frac{N_{c}^{2}}{192 \pi^{2}} \frac{Q^{2}}{\Lambda L} \ln ^{3} \frac{Q_{s}^{2}(x, 0)}{Q^{2}} \quad \text { and } \quad F_{\mathrm{L}}=\frac{N_{c}^{2}}{16 \pi^{2}} \frac{Q^{2}}{\Lambda L} \ln ^{2} \frac{Q_{s}^{2}(x, 0)}{Q^{2}}
$$

where we see again the extra logarithmic enhancement in the transverse structure function.

In what follows, we shall argue that the results in Eq. (4.42) have a natural physical interpretation in terms of parton saturation in the boundary gauge theory. Our discussion will closely follow that of the plasma structure functions at strong coupling, as originally presented in Ref. [34]. To that purpose, two more ingredients turn out to be extremely useful:

(a) The associated energy-momentum sum rules, which can be derived via the same strategy as in the dilaton case (recall the discussion of Eq. (3.50)). Namely, one first computes the leadingtwist, or single scattering, contributions to the Lorentz invariants $\Pi_{1}\left(x, Q^{2}\right)$ and $\Pi_{2}\left(x, Q^{2}\right)$, which are real quantities as anticipated:

$$
\Pi_{1}^{(1)}=\frac{N_{c}^{2}}{20} \frac{\Lambda^{2}}{x^{2}} \quad \text { and } \quad \Pi_{2}^{(1)}=\frac{11 N_{c}^{2}}{15} \frac{\Lambda^{2}}{Q^{2}}
$$

Then one uses analyticity and contour integration in the complex $\nu \equiv 1 / x$ plane to finally deduce

$$
\int_{0}^{1} \mathrm{~d} x x F_{1}\left(x, Q^{2}\right)=\frac{N_{c}^{2}}{80} \Lambda^{2} \quad \text { and } \quad \int_{0}^{1} \mathrm{~d} x F_{2}\left(x, Q^{2}\right)=\frac{11 N_{c}^{2}}{120} \Lambda^{2},
$$

which should more properly be understood as limiting values when $Q^{2} \rightarrow \infty$ (since, as in the dilaton case, we have neglected the higher-twist contributions to $\Pi\left(\nu, Q^{2}\right)$ near $\left.\nu=0\right)$. Examining Eqs. (4.41) and (4.42), we can verify that these sum rules are parametrically satisfied by our previous estimates for the structure functions, and that the integrals are dominated by $x \sim x_{s}$, as expected. In fact, by using the exact, integral, expressions for $\Pi^{i i}$ and $\Pi^{--}$derived in the previous subsection, one can explicitly evaluate the above integrals of $F_{1}$ and $F_{2}$ and thus check not only their high- $Q^{2}$ limit shown in Eq. (4.46), but also the subleading corrections at large $Q^{2}$. This will be detailed in Appendix D.

Once again, the sum-rules (4.46) extract the contribution of the energy-momentum tensor to the operator product expansion for the current-current correlator. In view of this and of the mass dimensions of the above integrals, it is natural to interpret them as the energy per unit length in the nucleus rest frame. 
(b) The expression of the gluon distribution at saturation in perturbative QCD, which reads, parametrically [6]

$$
\frac{x G\left(x, Q^{2}\right)}{\pi R^{2}} \sim \frac{1}{\lambda} N_{c}^{2} Q^{2} \ln \frac{Q_{s}^{2}(x)}{Q^{2}}
$$

where we now assume $\lambda \equiv g^{2} N_{c} \ll 1$ and $N_{c} \gg 1$. (The last assumption is not essential, but merely convenient for the sake of comparison with the AdS/CFT results.) In writing Eq. (4.47), we have also divided by the nuclear transverse area $\pi R^{2}$, so that the quantity shown there is the number of gluons per unit transverse area having transverse momenta $k_{\perp} \lesssim Q<Q_{s}(x)$. Then, the estimate in the r.h.s. of this equation can be understood as follows: the gluons with longitudinal momentum fraction $x$ and low transverse momenta $k_{\perp}<Q_{s}(x)$ are saturated, in the sense that there is a number of gluons of $\mathcal{O}(1 / \lambda)$ of each color per unit cell in the phasespace, and this number does not grow with the energy anymore (in contrast to what happens at larger momenta $k_{\perp}>Q_{s}(x)$ ). Specifically, Eq. (4.47) implies the following estimate for the gluon occupation number at saturation in QCD at weak coupling and large $N_{c}$ :

$$
\left.n\left(x, k_{\perp}\right) \equiv \frac{1}{N_{c}^{2} \pi R^{2}} \frac{\mathrm{d} x G\left(x, Q^{2}\right)}{\mathrm{d} Q^{2}}\right|_{Q=k_{\perp}} \sim \frac{1}{\lambda} \ln \frac{Q_{s}^{2}(x)}{k_{\perp}^{2}}
$$

which shows only a weak, logarithmic, dependence upon the energy, via the corresponding dependence of the saturation momentum: $Q_{s}^{2}(x) \sim 1 / x^{\omega}$ with $\omega \sim \mathcal{O}(\lambda)$.

We shall now argue that the gluon distribution in Eq. (4.47) is the natural quantity to compare with the structure function $F_{2}$ in Eq. (4.42) and that this comparison suggests a partonic interpretation for the latter which is further supported by the sum rules (4.46).

As already mentioned, the $\mathcal{R}$-current directly couples to fermionic and scalar fields of $\mathcal{N}=$ $4 \mathrm{SYM}$, so the respective structure function $F_{2}$ is most naturally related (at least at weak coupling, where the standard OPE arguments apply) to the parton distributions for these fields. However, the latter lie in the adjoint representation of the color group; hence, for the purposes of the $N_{c}$ power counting, it is more appropriate to compare $F_{2}$ in $\mathcal{N}=4 \mathrm{SYM}$ to the gluon distribution in QCD, and not to the respective structure function, which rather describes the distribution of quarks in the fundamental representation. Such a comparison, together with the formal similarity between Eq. (4.47) and the expression (4.42) for $F_{2}$ (they both show the same parametric dependencies upon $N_{c}^{2}$ and $Q^{2}$ ), immediately suggests that partons are saturated at strong coupling too, but with occupation numbers of order one, rather than $1 / \lambda$. More precisely, for $k_{\perp} \ll Q_{s}(x)$, Eq. (4.42) implies

$$
\left.n\left(x, k_{\perp}\right) \equiv \frac{\Lambda L}{N_{c}^{2}} \frac{\mathrm{d} F_{2}\left(x, Q^{2}\right)}{\mathrm{d} Q^{2}}\right|_{Q=k_{\perp}} \sim \ln \frac{Q_{s}^{2}(x)}{k_{\perp}^{2}},
$$

which suggests that, at strong coupling, the mechanism for parton saturation has the same effect as at weak coupling [6] (although its dynamical nature might be very different) — it limits the rate for parton emission with increasing rapidity $Y \equiv \ln (1 / x): \mathrm{d} n / \mathrm{d} Y \sim$ const. for $k_{\perp} \lesssim Q_{s}(Y)$. At least at weak coupling, this limitation is associated with the formation of a 'color glass condensate' [6] — a strong color mean field collectively created by all the small- $x$ partons, which blocks the radiation of new partons once the density is high enough. It is likely 
that a similar picture holds at strong coupling as well. But unlike at weak coupling, where this saturation requires parametrically large occupation numbers $n \sim 1 / \lambda \gg 1$, to compensate for the weakness of the coupling, at strong coupling it occurs already for occupation numbers of $\mathcal{O}(1)$, since the mutual repulsion becomes strong as soon as the partons are allowed to interact with each other.

In writing Eq. (4.49) we have extracted the parton occupation number at saturation out of the structure function $F_{2}$ in the same way as we would do at weak coupling. To further justify this identification, let us now show that it is also consistent with the sum rules (4.46), which in fact explain the additional factor $\Lambda L$ that we have inserted in the definition of $n\left(x, k_{\perp}\right)$ in Eq. (4.49). We have already mentioned below Eq. (4.46) that the following integral

$$
\int_{0}^{1} \mathrm{~d} x F_{2}\left(x, Q^{2}\right) \sim x_{s} F_{2}\left(x_{s}, Q^{2}\right) \sim N_{c}^{2} \Lambda^{2},
$$

is proportional to the energy density per unit longitudinal distance in the target rest frame. Using this, we would like to construct the energy per unit transverse area in the shockwave ${ }^{16}$. This is obtained by multiplying the quantity above by the longitudinal extent $L$ (to get the total energy), then dividing it by the transverse area (i.e. multiplying it by a factor $\Lambda^{2}$ ), and finally multiplying it by the Lorentz factor $\gamma$ (to make a boost to the target infinite momentum frame). One thus gets

$$
\frac{\mathrm{d} E_{\mathrm{sw}}}{\mathrm{d}^{2} b} \sim \gamma L \Lambda^{2}\left(x F_{2}\left(x, Q^{2}\right)\right)_{x=x_{s}} \sim k^{+}\left(\Lambda L F_{2}\left(x, Q^{2}\right)\right)_{x=x_{s}},
$$

where we have also identified the longitudinal momentum $k^{+}=x p^{+}=x \gamma \Lambda$ of the parton (scalar or adjoint fermion) which absorbs the virtual photon in this frame. The last estimate above suggests that, at least for $x \sim x_{s}$ where most of the energy is located, the quantity $\Lambda L F_{2}\left(x, Q^{2}\right)$ has the meaning of the total number of partons (with energy fraction $x$ ) per unit transverse area as probed on the resolution scale $Q^{2}$ :

$$
\Lambda L F_{2}\left(x, Q^{2}\right) \sim \int^{Q^{2}} \mathrm{~d}^{2} k_{\perp} \frac{\mathrm{d} n}{\mathrm{~d}^{2} k_{\perp} \mathrm{d}^{2} b_{\perp}} .
$$

This is indeed the same as Eq. (4.49) since, by definition, $n\left(x, k_{\perp}\right) \equiv\left(1 / N_{c}^{2}\right)\left[\mathrm{d} n / \mathrm{d}^{2} k_{\perp} \mathrm{d}^{2} b_{\perp}\right]$.

\section{Acknowledgments}

We would like to thank Guillaume Beuf for early discussions which motivated this work and also for pointing to us Refs. $[27,28]$. We are grateful to Al Mueller for many inspiring discussions and comments on the manuscript. We acknowledge useful discussions with Javier Albacete, Jorge Casalderrey-Solana and Tony Rebhan. The work of E.A. and E.I. is supported in part by Agence Nationale de la Recherche via the programme ANR-06-BLAN-0285-01. The research of L.M. is supported under DOE Contract No. DE- AC02-98CH10886. The work of D.N.T. is supported by the Austrian Science Foundation FWF project no. P19958 and in part by ÖAD project nos. FR 08/2009 and ES 12/2009.

\footnotetext{
${ }^{16}$ Of course, we already know the corresponding result - it reads $\left(\mathrm{d} E_{\mathrm{sw}} / \mathrm{d}^{2} b\right) \sim \gamma N_{c}^{2} \Lambda^{4} L-$, but here we are rather interested in expressing this result in terms of $F_{2}$ and thus deducing a partonic interpretation for the latter.
} 


\section{A. From black holes to shockwaves}

Let us modify the temperature dependent piece of the $\mathrm{AdS}_{5}$ black-brane metric by an arbitrary function depending on the $x^{3}$ coordinate. That is, using Fefferman-Graham coordinates, we consider the metric in

$$
\mathrm{d} s^{2}=\frac{1}{z^{2}}\left\{-\frac{(1-a)^{2}}{1+a} \mathrm{~d} t^{2}+(1+a)\left[\mathrm{d} x_{\perp}^{2}+\left(\mathrm{d} x^{3}\right)^{2}\right]+\mathrm{d} z^{2}\right\} \quad \text { with } \quad a \equiv c\left(x^{3}\right) \frac{z^{4}}{4 z_{0}^{4}} .
$$

Here $c\left(x^{3}\right)$ is a dimensionless function, which reduces to unity for the black-brane solution, and $z_{0}=1 / \pi T$ with $T$ the Hawking temperature. In general Eq. (A.1) is not a solution to the Einstein equations. Now we would like to make a boost and choose a frame $\dot{x}^{m}$ which moves with velocity $-v$ with respect to the frame $x^{m}$ and along their common axis in the 3-direction. In the new frame we would also like to switch to light-cone coordinates and it is straightforward to check that the combined transformation of coordinates reads

$$
\begin{aligned}
t & =\frac{1}{\sqrt{2} \gamma(1+v)} \dot{x}^{+}+\frac{\gamma(1+v)}{\sqrt{2}} \dot{x}^{-}, \\
x^{3} & =\frac{1}{\sqrt{2} \gamma(1+v)} \dot{x}^{+}-\frac{\gamma(1+v)}{\sqrt{2}} \dot{x}^{-} .
\end{aligned}
$$

Clearly the transverse and radial coordinates remain unaffected. The metric tensor transforms according to

$$
\dot{g}_{m n}=\frac{\partial x^{p}}{\partial \dot{x}^{m}} \frac{\partial x^{q}}{\partial \dot{x}^{n}} g_{p q},
$$

so that the components which are transformed read

$$
\begin{aligned}
& \dot{g}_{++}=\frac{1}{2 \gamma^{2}(1+v)^{2}}\left(g_{t t}+g_{33}\right)=\frac{2 a}{\gamma^{2}(1+v)^{2} z^{2}(1+a)}, \\
& \dot{g}_{--}=\frac{\gamma^{2}(1+v)^{2}}{2}\left(g_{t t}+g_{33}\right)=\frac{2 \gamma^{2}(1+v)^{2} a}{z^{2}(1+a)}, \\
& \dot{g}_{+-}=\dot{g}_{-+}=\frac{1}{2}\left(g_{t t}-g_{33}\right)=-\frac{\left(1+a^{2}\right)}{z^{2}(1+a)} .
\end{aligned}
$$

When the boost is ultra relativistic, that is for $\gamma \gg 1 \Leftrightarrow v \rightarrow 1$, we expect the metric to simplify. Indeed we have

$$
\dot{g}_{++} \simeq 0, \quad \dot{g}_{--} \simeq \frac{1}{z^{2}} \frac{2 \gamma^{2} \tilde{c}\left(\dot{x}^{-}\right) z^{4}}{z_{0}^{4}} \frac{1}{1+\frac{\tilde{c}\left(\hat{x}^{-}\right) z^{4}}{4 z_{0}^{4}}} \quad \text { and } \quad \dot{g}_{+-}=\dot{g}_{-+}=-\frac{1}{z^{2}} \frac{1+\frac{\left[\tilde{c}\left(\dot{x}^{-}\right)\right]^{2} z^{8}}{16 z_{0}^{8}}}{1+\frac{\tilde{c}\left(\dot{x}^{-}\right) z^{4}}{4 z_{0}^{4}}}
$$

where we have substituted $a$ with its explicit form and we have defined $\tilde{c}\left(\dot{x}^{-}\right)=c\left(x^{3}\right)$. Notice that in this ultra relativistic limit Eq. (A.3) simplifies to $x^{3} \simeq-\sqrt{2} \gamma x^{-}$. Furthermore, close to the boundary, i.e. when $z \ll z_{0}$, one can approximate $g_{i i} \simeq 1 / z^{2}$ in Eq. (A.1) and also neglect the last factor in $\dot{g}_{--}$and $\dot{g}_{+-}$in Eq. A.8). However, notice that $\dot{g}_{--}$does not vanish since it is enhanced by the square of the large boost factor. Putting everything together, and dropping the primes, we can write the metric as

$$
\mathrm{d} s^{2} \simeq \frac{1}{z^{2}}\left[\frac{2 \gamma^{2} \tilde{c}\left(x^{-}\right) z^{4}}{z_{0}^{4}}\left(\mathrm{~d} x^{-}\right)^{2}-2 \mathrm{~d} x^{+} \mathrm{d} x^{-}+\mathrm{d} x_{\perp}^{2}+\mathrm{d} z^{2}\right]
$$


This is formally the same as the metric corresponding to a homogeneous in the transverse space shockwave, cf. Eq. (2.6), which we recall is an exact solution to the Einstein equations. Here, however, this metric emerges only as a near-boundary approximation valid for $z \ll 1 / T$. On the other hand, the more general boosted-plasma metric in Eq. (A.8) keeps trace of the black hole horizon at $z=z_{0}$, and it is valid only for $z \leq z_{0}$. This makes it natural to supply the shockwave in Eq. (2.6) with a radial cutoff at finite $z \sim 1 / T$ or in general at $1 / \Lambda$, where $\Lambda$ is an infrared cutoff in the dual gauge theory.

Let us finally observe that when the function $c\left(x^{3}\right)$ has support only in an interval of finite length $L$, in the boosted system it will be transformed into a delta function. For instance, in the case of a slice of a plasma (in the dual gauge theory) with $c\left(x^{3}\right)=1$ for $\left|x^{3}\right| \leq L / 2$ and zero otherwise, in the boosted frame and in light cone coordinates we find

$$
\tilde{c}\left(x^{-}\right) \simeq \frac{L}{2 \sqrt{2} \gamma} \delta\left(x^{-}\right)
$$

\section{B. The bulk-to-bulk propagator}

In this Appendix we outline the construction of the Green's functions for the equations of motion. For definiteness, let us consider the dilaton case. Taking the Fourier transform of (3.25) w.r.t. $x^{-}$ and $x_{\perp}$ we have

$$
\left(\partial_{z}^{2}-\frac{3}{z} \partial_{z}-K^{2}\right) G\left(z, \dot{z} ; K^{2}\right)=z^{3} \delta(z-\dot{z})
$$

where we have defined the variable $K^{2} \equiv k_{\perp}^{2}-2 q^{-} k^{+}$. The solution to the eigenvalue problem for the operator $\partial_{z}^{2}-(3 / z) \partial_{z}$ is given by

$$
\left(\partial_{z}^{2}-\frac{3}{z} \partial_{z}\right) z^{2} \mathrm{~J}_{2}(\omega z)=-\omega^{2} z^{2} \mathrm{~J}_{2}(\omega z),
$$

and the eigenfunctions satisfy the completeness relation

$$
\int_{0}^{\infty} \mathrm{d} \omega \omega z^{2} \mathrm{~J}_{2}(\omega z) \dot{z}^{2} \mathrm{~J}_{2}(\omega \dot{z})=z^{3} \delta(z-\dot{z})
$$

Therefore we can write the Green's function as

$$
G\left(z, \dot{z} ; K^{2}\right)=-\int_{0}^{\infty} \mathrm{d} \omega \omega \frac{1}{\omega^{2}+K^{2}} z^{2} \mathrm{~J}_{2}(\omega z) \dot{z}^{2} \mathrm{~J}_{2}(\omega \dot{z}) .
$$

Now we would like to integrate over $\omega$ and we need to distinguish two possibilities according to the sign of $K^{2}$. In the spacelike case $K^{2}>0$ we find

$$
G\left(z, \dot{z} ; K^{2}>0\right)=-z^{2} \dot{z}^{2}\left[\mathrm{~K}_{2}(K \dot{z}) \mathrm{I}_{2}(K z) \Theta(\dot{z}-z)+\mathrm{K}_{2}(K z) \mathrm{I}_{2}(K \dot{z}) \Theta(z-\dot{z})\right] .
$$

In the timelike case $K^{2}<0$ we need a prescription to avoid hitting the pole which is located on the positive $\omega$-axis. Letting $K^{2} \rightarrow K^{2}-i \epsilon$ with $\epsilon>0$, we find (in agreement with the analytic continuation of $(\underline{B .5}))$

$$
G\left(z, \dot{z} ; K^{2}<0\right)=-\frac{i \pi z^{2} \dot{z}^{2}}{2}\left[\mathrm{H}_{2}^{(1)}(|K| \dot{z}) \mathrm{J}_{2}(|K| z) \Theta(\dot{z}-z)+\mathrm{H}_{2}^{(1)}(|K| z) \mathrm{J}_{2}(|K| \dot{z}) \Theta(z-\dot{z})\right] .(\mathrm{B} .
$$


Following the same procedure we can construct the Green's functions that we encountered for the $\mathcal{R}$-current in Sect. 4 . The transverse and longitudinal Green's functions in equations (4.23) and $(4.30)$ are given by

$$
\begin{aligned}
& G_{\mathrm{T}}\left(z, \dot{z} ; K^{2}>0\right)=-z \dot{z}\left[\mathrm{~K}_{1}(K \dot{z}) \mathrm{I}_{1}(K z) \Theta(\dot{z}-z)+\mathrm{K}_{1}(K z) \mathrm{I}_{1}(K \dot{z}) \Theta(z-\dot{z})\right], \\
& G_{\mathrm{L}}\left(z, \dot{z} ; K^{2}>0\right)=-z \dot{z}\left[\mathrm{~K}_{0}(K \dot{z}) \mathrm{I}_{0}(K z) \Theta(\dot{z}-z)+\mathrm{K}_{0}(K z) \mathrm{I}_{0}(K \dot{z}) \Theta(z-\dot{z})\right],
\end{aligned}
$$

respectively. The corresponding functions for $K^{2}<0$ are obtained via analytic continuation. Now the Green's functions in coordinate space will be given by

$$
G\left(z, \dot{z} ; x^{-}-y^{-}, x_{\perp}-y_{\perp}\right)=\int \frac{\mathrm{d} k^{+} \mathrm{d}^{2} k_{\perp}}{(2 \pi)^{3}} \mathrm{e}^{-i k^{+}\left(x^{-}-y^{-}\right)+i k_{\perp} \cdot\left(x_{\perp}-y_{\perp}\right)} G\left(z, \dot{z} ; K^{2}\right) .
$$

and the integration over $k^{+}$can be easily performed using the representation in Eq. (B.4) with our prescription $K^{2} \rightarrow K^{2}-i \epsilon$. The pole is at $\left(\omega^{2}+k_{\perp}^{2}\right) / 2 q^{-}-i \epsilon$ and is enclosed by the integration contour only for $x^{-}>y^{-}$. Thus the $k^{+}$-integration leads to the factor

$$
-\int \frac{\mathrm{d} k^{+}}{2 \pi} \frac{\mathrm{e}^{-i k^{+}\left(x^{-}-y^{-}\right)}}{\omega^{2}+k_{\perp}^{2}-2 q^{-} k^{+}-i \epsilon}=-\frac{i \Theta\left(x^{-}-y^{-}\right)}{2 q^{-}} \exp \left[-\frac{i\left(x^{-}-y^{-}\right)\left(\omega^{2}+k_{\perp}^{2}\right)}{2 q^{-}}\right],
$$

which in turn yields the expression in Eq. (3.26).

The bulk-to-bulk propagator has been already constructed in the past (see for example [16, $56,57]$ ), but mostly using Euclidean signature, or using standard Minkowski coordinates instead of light cone coordinates. Therefore, let us now show how the methods used in this paper lead to these known results. We will aim to write the propagator as a function of the chordal distance introduced earlier in Sect. 2. This property arises from the fact that the differential operator can be rewritten as the Laplacian of the hyperbolic space $\mathbb{H}_{d+1}$ with coordinates $\left(z, x_{1}, \ldots, x_{d}\right)$. We shall be interested in the case $d=4$. Defining the 4-dimensional vector $r=x-y$ and performing a Wick's rotation in Eq. (B.4), the Euclidean Green's function in coordinate space is obtained as (below, $k^{2}=\sum_{i=1}^{4} k_{i}^{2}$ is strictly positive)

$$
G(z, \dot{z} ; r)=-z^{2} \dot{z}^{2} \int \frac{\mathrm{d}^{4} k}{(2 \pi)^{4}} \mathrm{e}^{i k \cdot r} \int_{0}^{\infty} \mathrm{d} \omega \omega \frac{1}{\omega^{2}+k^{2}} \mathrm{~J}_{2}(\omega z) \mathrm{J}_{2}(\omega \dot{z}) .
$$

First we perform the integration over $k$. In $d$-dimensions we have

$$
\int \frac{\mathrm{d}^{d} k}{(2 \pi)^{d}} \frac{\mathrm{e}^{i k \cdot r}}{\omega^{2}+k^{2}}=\frac{1}{(2 \pi)^{d / 2}}\left(\frac{\omega}{r}\right)^{d / 2-1} \mathrm{~K}_{d / 2-1}(\omega r) .
$$

Using the above for $d=4$ and the identity $\mathrm{K}_{1}(\omega r)=-(1 / \omega) \partial_{r} \mathrm{~K}_{0}(\omega r)$, we can write the coordinate space propagator as

$$
G(z, \dot{z} ; r)=\frac{z^{2} \dot{z}^{2}}{4 \pi^{2} r} \frac{\partial}{\partial r} \int_{0}^{\infty} \mathrm{d} \omega \omega \mathrm{J}_{2}(\omega z) \mathrm{J}_{2}(\omega z) \mathrm{K}_{0}(\omega r)=\frac{z^{2} \dot{z}^{2}}{4 \pi^{2} r} \frac{\partial}{\partial r} \frac{\left(r_{2}-r_{1}\right)^{2}}{r_{1} r_{2}\left(r_{2}+r_{1}\right)^{2}}
$$

where we have found convenient to define

$$
r_{1}=\sqrt{r^{2}+(z-\dot{z})^{2}} \text { and } r_{2}=\sqrt{r^{2}+(z+\grave{z})^{2}} .
$$


Now we introduce a chordal coordinate $\xi$, which is closely related to the chordal distance $q$ defined in Sect. 2, according to

$$
\xi=\frac{2 z \dot{z}}{r^{2}+z^{2}+\dot{z}^{2}}=\frac{1}{1+2 q}
$$

It is straightforward to make a change of variable from $r$ to $\xi$ and performing the required algebraic manipulations we finally arrive at

$$
G(\xi)=-\frac{1}{8 \pi^{2}} \frac{\xi^{4}\left(1+2 \sqrt{1-\xi^{2}}\right)}{\left(1-\xi^{2}\right)^{3 / 2}\left(1+\sqrt{1-\xi^{2}}\right)^{2}}=-\frac{3 \xi^{4}}{32 \pi^{2}}{ }_{2} F_{1}\left(2,5 / 2,3 ; \xi^{2}\right)
$$

where ${ }_{2} F_{1}$ is the hypergeometric function. As announced, this expression depends on the variables $z, z$ and $r$ only through a single chordal coordinate. Up to a minus sign, which is due to different conventions, this is equal to the bulk-to-bulk propagator given in $[57]^{17}$.

Furthermore, let us choose the 'transverse' space to be 2-dimensional. By making use of Eq. (B.12) for $d=2$, we can write the propagator as

$$
G_{2}(z, \dot{z} ; r)=-\frac{z^{2} \dot{z}^{2}}{2 \pi r} \int_{0}^{\infty} \mathrm{d} \omega \omega \mathrm{J}_{2}(\omega z) \mathrm{J}_{2}(\omega \dot{z}) \mathrm{K}_{0}(\omega r)=\frac{z^{2} \dot{z}^{2}}{4 \pi^{2} r} \frac{\left(r_{2}-r_{1}\right)^{2}}{r_{1} r_{2}\left(r_{2}+r_{1}\right)^{2}}
$$

We can easily express the above in terms of the chordal distance $q$ to find

$$
G_{2}(z, \dot{z} ; q)=-\frac{z \dot{z}}{128 \pi q^{3}}{ }_{2} F_{1}(3,5 / 2,5 ;-1 / q) \text {. }
$$

Multiplying the above by $8 \pi^{2} E \delta\left(x^{-}\right) / N_{c}^{2}$, as dictated from Eqs. (2.18) and (2.4), we are lead to Eq. (2.19). Finally, yet another definition is to use $\cosh u=1 / \xi$, which means that we can write

$$
G_{2}(z, \dot{z} ; u)=-\frac{z \dot{z}}{4 \pi} \frac{\mathrm{e}^{-2 u}}{\sinh u}
$$

which, up to an overall factor independent of $u$, is the form used in $[16]^{18}$.

\section{Prefactor of the structure function}

In this short Appendix we give an alternative calculation of the structure function at saturation in the limit $Q^{2} \ll Q_{s}^{2}(x, 0)$, in the case of probing with a dilaton, and in order to determine the correct prefactor. We can write the most dominant contribution to the structure function as

$$
F\left(x, Q^{2}\right)=\frac{N_{c}^{2}}{32 \pi^{2}} \frac{Q^{6} \Lambda}{x L} \int_{0}^{R} \mathrm{~d}^{2} b_{\perp} \int_{0}^{\infty} \mathrm{d} z z \mathrm{~K}_{2}^{2}(Q z)\left\{1-\cos \left[Q^{2} Q_{s}^{2}\left(x, b_{\perp}\right) z^{4}\right]\right\}
$$

where the radius $R$ is defined by $Q_{s}^{2}(x, R)=Q^{2}$. We have already seen in Sect. 3.4 that the dominant contribution in this dilaton case comes from the region $z \ll 1 / Q$, thus it is natural to

\footnotetext{
${ }^{17}$ One needs to set $\Delta=d=4$ in Eq. (6.12) of that reference in order to obtain the 5-dimensional massless case.

${ }^{18} \mathrm{In}$ Eq. (25) in that reference, where the sign in the exponent should be negative.
} 
approximate the Bessel function by its lowest order term, that is $\mathrm{K}_{2}(Q z) \simeq 2 /(Q z)^{2}$. Then the $z$-integration gives $\sqrt{2 \pi} Q_{s}\left(x, b_{\perp}\right) / Q^{3}$ and the structure function becomes

$$
F\left(x, Q^{2}\right)=\frac{N_{c}^{2}}{16 \sqrt{2 \pi}} \frac{Q^{4}}{x \Lambda L} \frac{Q_{s}(x, 0)}{Q} \underbrace{\frac{\Lambda^{2}}{\pi} \int_{0}^{\infty} \mathrm{d}^{2} b_{\perp} \sqrt{\frac{f\left(b_{\perp} \Lambda\right)}{f(0)}}}_{\kappa} .
$$

Notice that we have replaced the radius $R$ in the upper limit of the impact parameter integration by $\infty$. This happens because the presence of the profile function in the numerator of the integrand effectively restricts the integration at values $b_{\perp} \lesssim 1 / \Lambda$, while at the same time $R \gg$ $1 / \Lambda$. A posteriori this also justifies the small- $z$ approximation since for all values of $b_{\perp}$ that contribute, one has $Q^{2} \ll Q_{s}^{2}\left(x, b_{\perp}\right)$. Now, $\kappa$ in Eq. (C.2) is a pure number which depends on the profile of the shockwave. For instance, $\kappa=8$ for the exponential type-I shockwave of the form $f\left(b_{\perp} \Lambda\right)=(1 / 2 \pi) \exp \left(-b_{\perp} \Lambda\right)$, while $\kappa=2$ for the type-II one.

\section{Explicit check of sum rules}

Here we will explicitly check the validity of the sum rule for the structure function $F_{1}$ as stated in the first expression in Eq. 4.46). One can follow a similar procedure for other sum rules. Using Eqs. (4.37) and (4.40) which provide us with $F_{1}$ and the scattering amplitude $\mathcal{T}$ respectively we have

$$
F_{1}\left(x, Q^{4}\right)=\frac{N_{c}^{2}}{32 \pi^{3}} \frac{Q^{4}}{x \Lambda L} \int_{0}^{\infty} \mathrm{d} z z \mathrm{~K}_{1}^{2}(Q z)\left[1-\cos \left(Q^{2} Q_{s}^{2} z^{4}\right)\right]
$$

We wish to integrate the above from $x=0$ to $x=1$ which is the physical region. Making a change of variables from $z$ to $\zeta=Q z$ and from $x$ to $t=Q_{s}^{2}(x) / Q^{2}$, we can write

$$
\int_{0}^{1} \mathrm{~d} x x F_{1}\left(x, Q^{2}\right)=\frac{N_{c}^{2} \Lambda^{2}}{64 \pi} \int_{0}^{\infty} \mathrm{d} \zeta \zeta \mathrm{K}_{1}^{2}(\zeta) \int_{t_{1}}^{\infty} \mathrm{d} t \frac{1-\cos t \zeta^{4}}{t^{2}}
$$

with $t_{1}=Q_{s}^{2}(1) / Q^{2}=\pi^{2} \Lambda^{3} L / 2 Q^{2}$. It is straightforward to see that the sum rule will be exactly recovered only when we set $t_{1} \rightarrow 0$ as the lower limit in the $t$-integration. For small but nonvanishing $t_{1}$, we can separate an integration from 0 to $\infty$ which gives the sum rule, minus an integration from 0 to $t_{1} \ll 1$ for which we can do a Taylor expansion. We find

$$
\int_{0}^{1} \mathrm{~d} x x F_{1}\left(x, Q^{2}\right)=\frac{N_{c}^{2} \Lambda^{2}}{80}-\frac{8 N_{c}^{2} \Lambda^{2}}{7 \pi} \frac{Q_{s}^{2}(1)}{Q^{2}}+\cdots .
$$

\section{References}

[1] M. Gyulassy and L. McLerran, New forms of QCD matter discovered at RHIC, Nucl. Phys. A750 (2005) 30-63, nucl-th/0405013.

[2] B. Muller, From Quark-Gluon Plasma to the Perfect Liquid, Acta Phys. Polon. B38 (2007) 3705-3730, 0710.3366.

[3] D. T. Son and A. O. Starinets, Viscosity, Black Holes, and Quantum Field Theory, Ann. Rev. Nucl. Part. Sci. 57 (2007) 95-118, 0704.0240]. 
[4] E. Iancu, Partons and jets in a strongly-coupled plasma from AdS/CFT, Acta Phys. Polon. B39 (2008) 3213-3280, arXiv:0812.0500].

[5] S. S. Gubser, S. S. Pufu, F. D. Rocha, and A. Yarom, Energy loss in a strongly coupled thermal medium and the gauge-string duality, arXiv:0902.4041.

[6] A.H. Mueller, Parton Saturation-An Overview, hep-ph/0111244; E. Iancu, A. Leonidov and L. McLerran, The Colour Glass Condensate: An Introduction, hep-ph/0202270. Published in QCD Perspectives on Hot and Dense Matter, Eds. J.-P. Blaizot and E. Iancu, NATO Science Series, Kluwer, 2002.

[7] J. M. Maldacena, The large N limit of superconformal field theories and supergravity, Adv. Theor. Math. Phys. 2 (1998) 231-252, hep-th/9711200.

[8] S. S. Gubser, I. R. Klebanov, and A. M. Polyakov, Gauge theory correlators from non-critical string theory, Phys. Lett. B428 (1998) 105-114, hep-th/9802109].

[9] E. Witten, Anti-de Sitter space and holography, Adv. Theor. Math. Phys. 2 (1998) 253-291, hep-th/9802150.

[10] M. Cheng et. al., The QCD Equation of State with almost Physical Quark Masses, Phys. Rev. D77 (2008) 014511, arXiv:0710.0354.

[11] E. Witten, Anti-de Sitter space, thermal phase transition, and confinement in gauge theories, Adv. Theor. Math. Phys. 2 (1998) 505-532, hep-th/9803131.

[12] R. A. Janik and R. B. Peschanski, Asymptotic perfect fluid dynamics as a consequence of AdS/CFT, Phys. Rev. D73 (2006) 045013, hep-th/0512162].

[13] D. Grumiller and P. Romatschke, On the collision of two shock waves in AdS5, JHEP 08 (2008) 027, arXiv:0803.3226.

[14] S. S. Gubser, S. S. Pufu, and A. Yarom, Entropy production in collisions of gravitational shock waves and of heavy ions, Phys. Rev. D78 (2008) 066014, arXiv:0805.1551.

[15] L. Alvarez-Gaume, C. Gomez, A. Sabio Vera, A. Tavanfar, and M. A. Vazquez-Mozo, Critical formation of trapped surfaces in the collision of gravitational shock waves, JHEP 02 (2009) 009, arXiv:0811.3969.

[16] S. Lin and E. Shuryak, Grazing Collisions of Gravitational Shock Waves and Entropy Production in Heavy Ion Collision, arXiv:0902.1508.

[17] S. S. Gubser, S. S. Pufu, and A. Yarom, Off-center collisions in AdS $S_{5}$ with applications to multiplicity estimates in heavy-ion collisions, arXiv:0902.4062.

[18] P. Figueras, V. E. Hubeny, M. Rangamani, and S. F. Ross, Dynamical black holes and expanding plasmas, arXiv:0902.4696.

[19] P. M. Chesler and L. G. Yaffe, Horizon formation and far-from-equilibrium isotropization in supersymmetric Yang-Mills plasma, Phys. Rev. Lett. 102 (2009) 211601, arXiv:0812.2053.

[20] P. M. Chesler and L. G. Yaffe, Boost invariant flow, black hole formation, and far-fromequilibrium dynamics in $N=4$ supersymmetric Yang-Mills theory, arXiv:0906.4426.

[21] G. Beuf, M. P. Heller, R. A. Janik, and R. Peschanski, Boost-invariant early time dynamics from $A d S / C F T$, arXiv:0906.4423.

[22] J. Polchinski and M. J. Strassler, The string dual of a confining four-dimensional gauge theory, hep-th/0003136. 
[23] J. Polchinski and M. J. Strassler, Hard scattering and gauge/string duality, Phys. Rev. Lett. 88 (2002) 031601, hep-th/0109174.

[24] J. Erlich, E. Katz, D. T. Son, and M. A. Stephanov, QCD and a Holographic Model of Hadrons, Phys. Rev. Lett. 95 (2005) 261602, hep-ph/0501128.

[25] A. Karch, E. Katz, D. T. Son, and M. A. Stephanov, Linear Confinement and AdS/QCD, Phys. Rev. D74 (2006) 015005, hep-ph/0602229.

[26] S. J. Brodsky and G. F. de Teramond, AdS/CFT and Light-Front QCD, p802.0514.

[27] J. Podolsky, Interpretation of the Siklos solutions as exact gravitational waves in the anti-de Sitter universe, Class. Quant. Grav. 15 (1998) 719-733, gr-qc/9801052].

[28] V. Kaigorodov Sov. Phys. Doklady. 7 (1963) 893.

[29] S. W. Hawking and G. F. R. Ellis, The Large scale structure of space-time, . Cambridge University Press, Cambridge, 1973.

[30] G. Beuf, Gravity dual of N=4 SYM theory with fast moving sources, arXiv:0903.1047.

[31] L. Susskind and E. Witten, The holographic bound in anti-de Sitter space, hep-th/9805114.

[32] A. W. Peet and J. Polchinski, UV/IR relations in AdS dynamics, Phys. Rev. D59 (1999) 065011, hep-th/9809022.

[33] J. Polchinski and M. J. Strassler, Deep inelastic scattering and gauge/string duality, JHEP 05 (2003) 012, hep-th/0209211.

[34] Y. Hatta, E. Iancu, and A. H. Mueller, Deep inelastic scattering off a N=4 SYM plasma at strong coupling, JHEP 01 (2008) 063, 0710.5297.

[35] Y. Hatta, E. Iancu, and A. H. Mueller, Jet evolution in the N=4 SYM plasma at strong coupling, JHEP 05 (2008) 037, arXiv:0803.2481.

[36] Y. Hatta, E. Iancu, and A. H. Mueller, Deep inelastic scattering at strong coupling from gauge/string duality : the saturation line, JHEP 01 (2008) 026, [0710.2148].

[37] C. A. Ballon Bayona, H. Boschi-Filho, and N. R. F. Braga, Deep inelastic scattering from gauge string duality in the soft wall model, JHEP 03 (2008) 064, arXiv:0711.0221.

[38] C. A. Ballon Bayona, H. Boschi-Filho, and N. R. F. Braga, Deep inelastic structure functions from supergravity at small $x$, JHEP 10 (2008) 088, arXiv:0712.3530.

[39] J. L. Albacete, Y. V. Kovchegov, and A. Taliotis, DIS on a Large Nucleus in AdS/CFT, JHEP 07 (2008) 074, arXiv:0806.1484.

[40] A. H. Mueller, A. I. Shoshi, and B.-W. Xiao, Deep inelastic and dipole scattering on finite length hot $\mathcal{N}=4$ SYM matter, Nucl. Phys. A822 (2009) 20-40, arXiv:0812.2897.

[41] S. Lin and E. Shuryak, Toward the AdS/CFT Gravity Dual for High Energy Collisions: II. The Stress Tensor on the Boundary, Phys. Rev. D77 (2008) 085014, arXiv:0711.0736.

[42] M. J. Strassler, Why Unparticle Models with Mass Gaps are Examples of Hidden Valleys, arXiv:0801.0629.

[43] D. M. Hofman and J. Maldacena, Conformal collider physics: Energy and charge correlations, 0803.1467 .

[44] L. Cornalba, M. S. Costa, and J. Penedones, Eikonal Approximation in AdS/CFT: Resumming the Gravitational Loop Expansion, JHEP 09 (2007) 037, arXiv:0707.0120. 
[45] R. C. Brower, M. J. Strassler, and C.-I. Tan, On The Pomeron at Large 't Hooft Coupling, JHEP 03 (2009) 092, arXiv:0710.4378.

[46] E. Levin, J. Miller, B. Z. Kopeliovich, and I. Schmidt, Glauber - Gribov approach for DIS on nuclei in $N=4 S Y M$, JHEP 02 (2009) 048, arXiv:0811.3586.

[47] M. E. Peskin and D. V. Schroeder, An Introduction to quantum field theory, . Reading, USA: Addison-Wesley (1995) 842 p.

[48] S. de Haro, S. N. Solodukhin, and K. Skenderis, Holographic reconstruction of spacetime and renormalization in the AdS/CFT correspondence, Commun. Math. Phys. 217 (2001) 595-622, hep-th/0002230.

[49] K. Skenderis, Lecture notes on holographic renormalization, Class. Quant. Grav. 19 (2002) 5849-5876, hep-th/0209067.

[50] L. D. McLerran and R. Venugopalan, Fock space distributions, structure functions, higher twists and small x, Phys. Rev. D59 (1999) 094002, hep-ph/9809427].

[51] D. T. Son and A. O. Starinets, Minkowski-space correlators in AdS/CFT correspondence: Recipe and applications, JHEP 09 (2002) 042, hep-th/0205051.

[52] C. P. Herzog and D. T. Son, Schwinger-Keldysh propagators from AdS/CFT correspondence, JHEP 03 (2003) 046, hep-th/0212072.

[53] S. S. Gubser, I. R. Klebanov, and A. M. Polyakov, A semi-classical limit of the gauge/string correspondence, Nucl. Phys. B636 (2002) 99-114, hep-th/0204051.

[54] A. V. Kotikov, L. N. Lipatov, A. I. Onishchenko, and V. N. Velizhanin, Three-loop universal anomalous dimension of the Wilson operators in $N=4$ SUSY Yang-Mills model, Phys. Lett. B595 (2004) 521-529, hep-th/0404092.

[55] R. C. Brower, J. Polchinski, M. J. Strassler, and C.-I. Tan, The Pomeron and Gauge/String Duality, JHEP 12 (2007) 005, hep-th/0603115.

[56] U. H. Danielsson, E. Keski-Vakkuri, and M. Kruczenski, Vacua, Propagators, and Holographic Probes in AdS/CFT, JHEP 01 (1999) 002, hep-th/9812007.

[57] E. D'Hoker and D. Z. Freedman, Supersymmetric gauge theories and the AdS/CFT correspondence, hep-th/0201253. 